\title{
Imprints on CMB Angular Power Spectrum Modes from Cosmological Reionization
}

\author{
Tiziana Trombetti ${ }^{1,2}$, Carlo Burigana ${ }^{1,3}$ \\ ${ }^{1}$ Istituto di Astrofisica Spaziale e Fisica Cosmica di Bologna, Bologna, Italy \\ ${ }^{2}$ Dipartimento di Fisica, Università La Sapienza, Roma, Italy \\ ${ }^{3}$ Dipartimento di Fisica e Scienze della Terra, Università degli Studi di Ferrara, Ferrara, Italy \\ Email: trombetti@iasfbo.inaf.it, burigana@iasfbo.inaf.it
}

Received September 11, 2012; revised October 15, 2012; accepted October 27, 2012

\begin{abstract}
The accurate understanding of the ionization history of the Universe plays a fundamental role in modern cosmology. It includes a phase of cosmological reionization after the standard recombination epoch, possibly associated to the early stages of structure and star formation. While the simple " $\tau$-parametrization" of the reionization process and, in particular, of its imprints on the Cosmic Microwave Background (CMB) anisotropy likely represents a sufficiently accurate modelling for the interpretation of current $\mathrm{CMB}$ data, a great attention has been recently posed on the accurate computation of the reionization signatures in the CMB for a large variety of astrophysical scenarios and physical processes. The amplitude and shape of the B-mode Angular Power Spectrum (APS) depends, in particular, on the tensortoscalar ratio, $r$, related to the energy scale of inflation, and on the reionization history, thus an accurate modeling of the reionization process will have implications for the precise determination of $r$ or to set more precise constraints on it through the joint analysis of $\mathrm{E}$ and B-mode polarization data available in the next future and from a mission of next generation. In this work we review some classes of astrophysical and phenomenological reionization histories, beyond the simple $\tau$-parametrization, a present a careful characterization of the imprints introduced in all the CMB APS modes. We have implemented a modified version of CAMB, the Cosmological Boltzmann code for computing the CMB anisotropy APS, to introduce the predicted hydrogen and helium ionization fractions. We compared the results obtained for these models for all the non-vanishing (in the assumed scenarios) modes of the CMB APS. Considering also the limitation from potential residuals of astrophysical foregrounds, we discussed the capability of next data to disentangle between different reionization scenarios in a wide range of tensor-to-scalar ratios.
\end{abstract}

Keywords: Cosmic Microwave Background Radiation: CMBR Polarization; Reionization; Gravitational Waves; CMBR Polarization

\section{Introduction}

The accurate understanding of the ionization history of the Universe plays a fundamental role in modern cosmology. The classical theory of hydrogen recombination for pure baryonic cosmological models [1,2] has been subsequently extended to non-baryonic dark matter models [3-5] and recently accurately updated to include also helium recombination in the current cosmological scenario (see e.g. [6] and references therein). Various models of the subsequent Universe ionization history have been considered to take into account additional sources of photon and energy production, possibly associated to the early stages of structure and star formation, able to significantly increase the free electron fraction, $x_{e}$, above the residual fraction $\left(\sim 10^{-3}\right)$ after the standard recombination epoch at $Z_{\text {rec }} \simeq 10^{3}$. These photon and energy production processes associated to the cosmo- logical reionization phase may leave imprints in the CMB providing a crucial "integrated" information on the so-called dark and dawn ages, i.e. the epochs before or at the beginning the formation of first cosmological structures. For this reason, among the extraordinary results achieved by the WMAP mission ${ }^{1}$, the contribution to the understanding of the cosmological reionization process has received a great attention.

This work is aimed at a careful characterization of the imprints introduced in the polarization anisotropy, with particular attention to the B-modes. The amplitude of the B-mode APS, whose detection represents a crucial indirect probe of the stochastic field of primordial gravity waves (see e.g. [7,8]; see also [9-11]), crucially depends on the ratio of the amplitudes of primordial tensor and scalar perturbations $r=T / S$, related to the energy scale

${ }^{1}$ http://lambda.gsfc.nasa.gov/product/map/current/. 
of inflation. On the other hand, the reionization history of the Universe leaves imprints on the shape of the B-mode as function of the multipole $\ell$ and on its amplitude. Thus, an accurate modeling of the reionization process will have implications for the precise determination of $r$ or to set more precise constraints on it through the joint analysis of all the CMB APS modes coming from data available in the next future and from a mission of next generation.

In Section 2 we briefly summarize the current observational information coming from WMAP on the cosmological reionization and describe its main imprints in the CMB. In Sections 3 and 4 we review the main properties of the two classes of reionization models considered in this work, astrophysical and phenomenological, respectively. For the latter kind of models, we explicitly provide the relationship between model parameters and the photon number and energy density injection. Section 5 concerns the numerical implementation we carried out to include the considered reionization scenarios in our Boltzmann code modified version. The experimental sensitivity of on-going and future CMB anisotropy space missions and the limitation coming from astrophysical foregrounds are discussed in Section 6, while our main results are presented in Section 7. Two Appendices (A and $\mathrm{B}$ ) report on some technical details of this work. A list of acronyms widely used in this paper can be found in Appendix C. Finally, in Section 8 we draw our conclusions.

\section{Cosmological Reionization}

To first approximation, the beginning of the reionization process is identified by the Thomson optical depth, $\tau$. The values of $\tau$ compatible with WMAP $3 \mathrm{yr}$ data, possibly complemented with external data, are typically in the range $\sim 0.06-0.12$ (corresponding to a reionization redshift in the range $\sim 8.5-13.5$ for a sudden reionization history), the exact interval depending on the considered cosmological model and combination of data sets [12]. Subsequent WMAP data releases improved the measure of $\tau$, achieving a $68 \%$ uncertainty of $\simeq \pm 0.015$ [1316]. Under various hypotheses (simple $\Lambda \mathrm{CDM}$ model with six parameters, inclusion of curvature and dark energy, of different kinds of isocurvature modes, of neutrino properties, of primordial helium mass fraction, or of a reionization width) the best fit of $\tau$ lies in the range $\simeq 0.086-0.089$, while allowing for the presence of primordial tensor perturbations or (and) of a running in the power spectrum of primordial perturbations the best fit of $\tau$ lies in the range to $\simeq 0.091-0.092(0.096)$. While this simple " $\tau$-parametrization" of the reionization process and, in particular, of its imprints on the CMB anisotropy likely represents a sufficiently accurate modelling for the interpretation of current CMB data, a great attention has been recently posed on the accurate com- putation of the reionization signatures in the CMB for a large variety of astrophysical scenarios and physical processes (see e.g. [17-25]) also in the view of WMAP accumulating data and of forthcoming and future experiments beyond WMAP (see [26] for a review). In [27] a detailed study of the impact of reionization, and the associated radiative feedback, on galaxy formation and of the corresponding detectable signatures has been presented, focussing on a detailed comparison of two well defined alternative prescriptions (suppression and filtering) for the radiative feedback mechanism suppressing star formation in small-mass halos, showing that they are consistent with a wide set of existing observational data but predict different $21 \mathrm{~cm}$ background signals accessible to future observations. The corresponding signatures detectable in the CMB have been then computed in [28].

Different scenarios have been investigated in [29] assuming that structure formation and/or extra sources of energy injection in the cosmic plasma can induce a double reionization epoch of the Universe at low (late processes) or high (early processes) redshifts, providing suitable analytical representations, called hereafter as "phenomenological reionization histories". In the late models, hydrogen was typically considered firstly ionized at a higher redshift ( $z \sim 15$, mimicking a possible effect by Pop III stars) and then at lower redshifts $(\mathrm{z} \sim 6$, mimicking the effect by stars in galaxies), while in the early reionization framework the authors hypothesized a peak like reionization induced by energy injection in the cosmic plasma at $\mathrm{z} \gtrsim$ some $\times 10^{2}$.

The sensitivity improvement of CMB polarization experiments calls for a complete and accurate characterization of all CMB APS, including the polarization B-modes, from low to high multipoles. Among the various studies, the reader could refer to the classical works by [30] and [31] for the connection of B-modes with inflation and their detectability, by [32] for the analysis of the kinetic Sunyaev-Zel'dovich effect from mildly non-linear largescale structure, and by [33] for the computation of B-modes induced by lensing.

In this work we review the relatively wide sets of astrophysical and phenomenological models introduced above and present detailed computations of the signatures they induce in the CMB temperature and polarization anisotropies. The methods described here can be also used as guidelines for the implementation of any other reionization scenario (see e.g. [34]). In particular, we present precise computations of the polarization B-mode APS for these models and compare them with the sensitivity of on-going (Planck ${ }^{2}$, see [35]) and future (assuming $\mathrm{COrE}^{3}$, see [36], as a reference) $\mathrm{CMB}$ space missions.

\footnotetext{
${ }^{2}$ www.rssd.esa.int/Planck.

${ }^{3} \mathrm{http}: / /$ www.core-mission.org/.
} 


\section{Signatures in the CMB}

The cosmological reionization leaves imprints on the CMB depending on the (coupled) ionization and thermal history. They can be divided in three categories ${ }^{4}: 1$ ) generation of CMB Comptonization and free-free spectral distortions associated to the IGM electron temperature increase during the reionization epoch [39-43], 2) suppression of CMB temperature anisotropies at large multipoles, $\ell$, due to photon diffusion, and 3 ) increasing of the power of CMB polarization and temperature-polarization cross-correlation anisotropy at various multipole ranges, mainly depending on the reionization epoch, because of the delay of the effective last scattering surface. The imprints on CMB anisotropies are mainly dependent on the ionization history while CMB spectral distortions strongly depend also on the thermal history. The reionization process mainly influences the polarization $\mathrm{E}$ and $\mathrm{B}$ modes, and the cross-correlation temperature-polarization mode because of the linear polarization induced by the Thomson scattering. The effect is typically particular prominent at low multipoles $\ell$, showing as a bump in the power spectra, otherwise missing.

Through this note we assume a flat $\Lambda \mathrm{CDM}$ model compatible with WMAP, described by matter and cosmological constant (or dark energy) density parameters $\Omega_{m}=0.24$ and $\Omega_{\Lambda}=0.76$, reduced Hubble constant $h=H_{0} /(100 \mathrm{~km} / \mathrm{s} / \mathrm{Mpc})=0.73$, baryon density

$\Omega_{b} h^{2}=0.022$, density contrast $\sigma_{8}=0.74$, and adiabatic scalar perturbations (without running) with spectral index $n_{s}=0.95$. We adopt a CMB background temperature of $2.725 \mathrm{~K}$ [44].

\section{Astrophysical Reionization Models}

The analysis of Ly $\alpha$ absorption in the spectra of the 19 highest redshift SDSS QSO shows a strong evolution of the Gunn-Peterson Ly $\alpha$ opacity at $z \sim 6 \quad[45,46]$. The downward revision of the electron scattering optical depth to $\tau=0.09 \pm 0.03$ in the release of the $3 \mathrm{yr}$ WMAP data, confirmed by subsequent releases, is consistent with "minimal reionization models" which do not require the presence of very massive $\left(M>100 M_{\odot}\right)$ Pop III stars [47]. The above models can be then used to explore the effects of reionization on galaxy formation, referred to as "radiative feedback".

A semi-analytic model to jointly study cosmic reionization and the thermal history of the IGM has been developed in [48]. According to Schneider and collaborators, the semi-analytical model developed by Choudhury \& Ferrara, complemented by the additional physics in${ }^{4}$ Inhomogeneous reionization also produces $\mathrm{CMB}$ secondary anisotropies that dominate over the primary CMB component for $l \gtrsim 4000$ and can be detected by upcoming experiments, like the ACT or ALMA $[37,38]$. troduced in [49], involves: 1) the treatment of IGM inhomogeneities by adopting the procedure of [50];2) the modelling of the IGM treated as a multiphase medium, following the thermal and ionization histories of neutral, HII, and HeIII regions simultaneously in the presence of ionizing photon sources represented by Pop III stars with a standard Salpeter IMF extending in the range 1 $100 M_{e}$ [51], Pop II stars with $Z=0.2 Z_{\odot}$ and Salpeter IMF, and QSO (particularly relevant at $z \lesssim 6$ ); 3 ) the chemical feedback controlling the prolonged transition from Pop III to Pop II stars in the merger-tree model by Schneider; 4) assumptions on the escape fractions of ionizing photons, considered to be independent of the galaxy mass and redshift, but scaled to the amount of produced ionizing photons. It then accounts for radiative feedback inhibiting star formation in low-mass galaxies. This semi-analytical model is determined by only four free parameters: the star formation efficiencies of Pop II and Pop III stars, a parameter, $\eta_{\text {esc }}$, related to the escape fraction of ionizing photons emitted by Pop II and Pop III stars, and the normalization of the photon mean free path, $\lambda_{0}$, set to reproduce low-redshift observations of Lyman-limit systems.

A variety of feedback mechanisms can suppress star formation in mini-halos, i.e. halos with virial temperatures $T_{\text {vir }}<10^{4} \mathrm{~K}$, particularly if their clustering is taken into account [52]. It is then possible to assume that stars can form in halos down to a virial temperature of $10^{4} \mathrm{~K}$, consistent with the interpretation of WMAP data [53] (but see also [54]). Even galaxies with virial temperature $T_{\text {vir }} \gtrsim 10^{4} \mathrm{~K}$ can be significantly affected by radiative feedback during the reionization process, as the increase in temperature of the cosmic gas can dramatically suppress their formation.

Based on cosmological simulations of reionization, [55] developed an accurate characterization of the radiative feedback on low-mass galaxies. This study shows that the effect of photoionization is controlled by a single mass scale in both the linear and non-linear regimes. The gas fraction within dark matter halos at any given moment is fully specified by the current filtering mass, which directly corresponds to the length scale over which baryonic perturbations are smoothed in linear theory. The results of this study provide a quantitative description of radiative feedback, independently of whether this is physically associated to photoevaporative flows or due to accretion suppression.

Two specific alternative prescriptions for the radiative feedback by these halos have been considered: 1) suppression model - in photoionized regions halos can form stars only if their circular velocity exceeds the critical value $v_{\text {crit }}=\left[2 k_{B} T / \mu m_{\mathrm{p}}\right]^{1 / 2}$; here $\mu$ is the mean molecular weight, $m_{p}$ is the proton mass, and $T$ is the average temperature of ionized regions, computed self- 
consistently from the multiphase IGM model; 2) filtering model - the average baryonic mass $M_{b}$ within halos in photoionized regions is a fraction of the universal value $f_{b}=\Omega_{b} / \Omega_{m}$, given by the fitting formula

$$
M_{b} / M=f_{b} /\left[1+\left(2^{1 / 3}-1\right) M_{\mathrm{C}} / M\right]^{3},
$$

where $M$ is the total halo mass and $M_{\mathrm{C}}$ is the total mass of halos that on average retain $50 \%$ of their gas mass. A good approximation for $M_{\mathrm{C}}$ is given by the linear-theory filtering mass,

$$
M_{\mathrm{F}}^{2 / 3}=(3 / a) \int_{0}^{a} \mathrm{~d} a^{\prime} M_{\mathrm{J}}^{2 / 3}\left(a^{\prime}\right)\left[1-\left(a^{\prime} / a\right)^{1 / 2}\right],
$$

where $a$ is the cosmic scale factor,

$$
M_{\mathrm{J}} \equiv(4 \pi / 3) \bar{\rho}\left(\pi c_{\mathrm{s}}^{2} / G \bar{\rho}\right)^{3 / 2}
$$

is the Jeans mass, $\bar{\rho}$ is the average total mass density of the Universe, and $C_{\mathrm{s}}$ is the gas sound speed.

The model free parameters are constrained by a wide range of observational data. Schneider and collaborators reported the best fit choice of the above four parameters for these two models that well accomplish a wide set of astronomical observations, such as the redshift evolution of Lyman-limit absorption systems, the Gunn-Peterson and electron scattering optical depths, the cosmic star formation history, and number counts of high redshift sources in the NICMOS Hubble Ultra Deep Field.

The two feedback prescriptions have a noticeable impact on the overall reionization history and the relative contribution of different ionizing sources. In fact, although the two models predict similar global star formation histories dominated by Pop II stars, the Pop III star formation rates have markedly different redshift evolution. Chemical feedback forces Pop III stars to live preferentially in the smallest, quasi-unpolluted halos (virial temperature $\gtrsim 10^{4} \mathrm{~K}$ ), which are those most affected by radiative feedback. In the suppression model, where star formation is totally suppressed below $v_{\text {crit }}$, Pop III stars disappear at $z \sim 6$; conversely, in the filtering model, where halos suffer a gradual reduction of the available gas mass, Pop III stars continue to form at $z \lesssim 6$, though with a declining rate. Since the star formation and photoionization rate at these redshifts are observationally well constrained, the star formation efficiency and escape fraction of Pop III stars need to be lower in the filtering model in order to match the data. Therefore reionization starts at $z \lesssim 15$ in the filtering model and only $16 \%$ of the volume is reionized at $z=10$ (while reionization starts at $Z \sim 20$ in the suppression model and it is $85 \%$ complete by $z=10$ ). For $6<z<7$, QSOs, Pop II and Pop III give a comparable contribution to the total photo-ionization rate in the filtering model, whereas in the suppression model reionization at $z<7$ is driven primarily by QSOs, with a smaller contribution from Pop II stars only.

The predicted free electron fraction and gas temperature evolution in the redshift range $7<z<20$ is very different for the two feedback models. In particular, in the filtering model the gas kinetic temperature is heated above the CMB value only at $z \lesssim 15$.

The Thomson optical depth, $\tau=\int \chi_{e} n_{e} \sigma_{T} c \mathrm{~d} t$, can be directly computed for the assumed $\Lambda$ CDM cosmological model parameters given the ionization histories: $\tau_{C F 06} \simeq 0.1017$ and $\tau_{G 00} \simeq 0.0631$ for the suppression and the filtering model, respectively. Note that these values are consistent with the Thomson optical depth range derived from WMAP $3 \mathrm{yr}$ (7 yr) data but with $\sim 1 \sigma(\sim 2 \sigma)$ difference among the two models, leaving a chance of accurately probing them with forthcoming CMB anisotropy experiments.

\section{Fitting Astrophysical Histories}

Since filtering and suppression models gives the redshift evolution of ionization fraction and electron temperature in a tabular way, as first step we derived these quantities with appropriate analytical functions, by means of a specific fitting tool, Igor Pro (v. 6.21) [56]. In order to have an accurate parametrization we divided the redshift in bins such to minimize the $\chi^{2}$ test given by the fit itself (see Appendix A for details).

The results found using the complete set of functions specific to each epoch of interest are plotted in Figure 1 for the ionization fraction and in Figure 2 for the electron temperature. Every graph shows the fitting functions (dashed red line) and the corresponding reionization model (solid black line).

The accuracy of the fit can be analyzed by means of the percentile difference among theoretical data and fitting functions, namely the ratios between the derived functions and the models data, as shown in Figure 3. From these plots one can see that the difference is always $<1 \%$, a bit greater in the filtering model but, in any case, the precision of the fit is always very high.

\section{Phenomenological Reionization Models}

We investigated two phenomenological double peaked reionization models introduced by Naselsky \& Chiang in which the Universe was reionized twice at different epochs, the first one, late reionization model, at $\mathrm{Z} \sim 10$ by Pop III stars and then at $Z \sim 6$ by stars in large galaxies, and the second one, early reionization model, at very high redshift $(z \gtrsim 100)$, induced for example by decay of unstable particles, followed by reionization mechanisms at $z \sim 6$ as predicted in the standard 

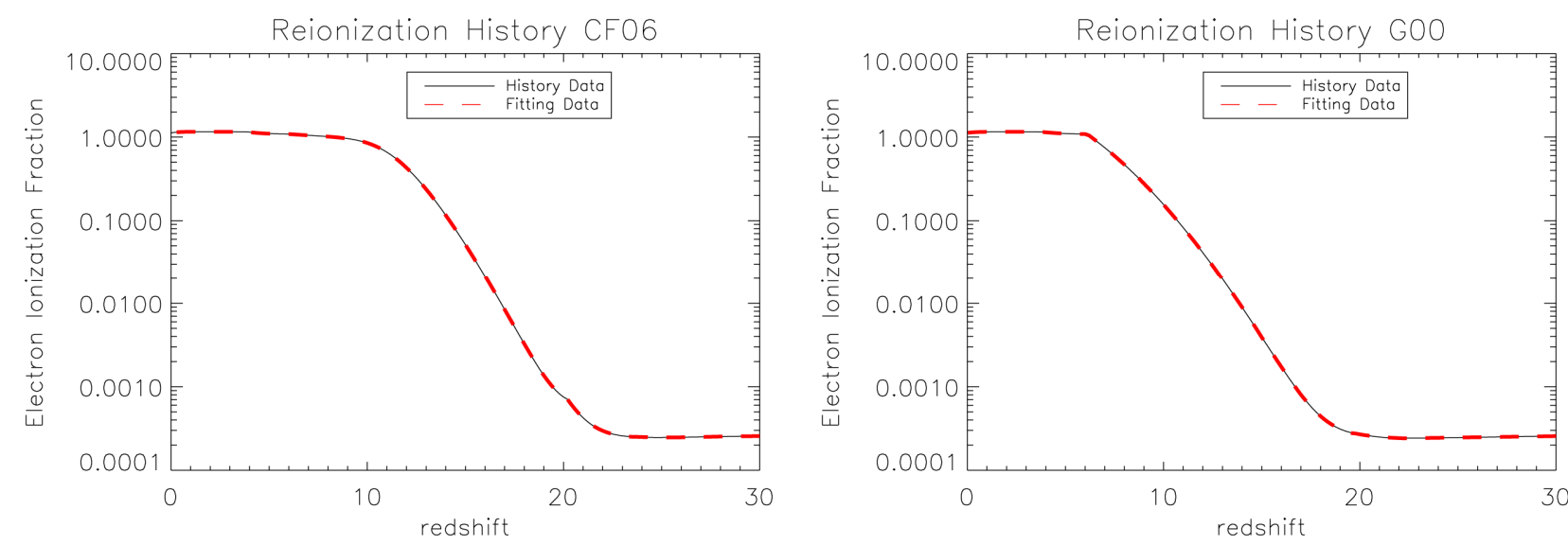

Figure 1. Ionization fraction: comparison between the tabulated data (solid black line) and the fit (dashed red line) for the suppression (left panel) and filtering model (right panel).
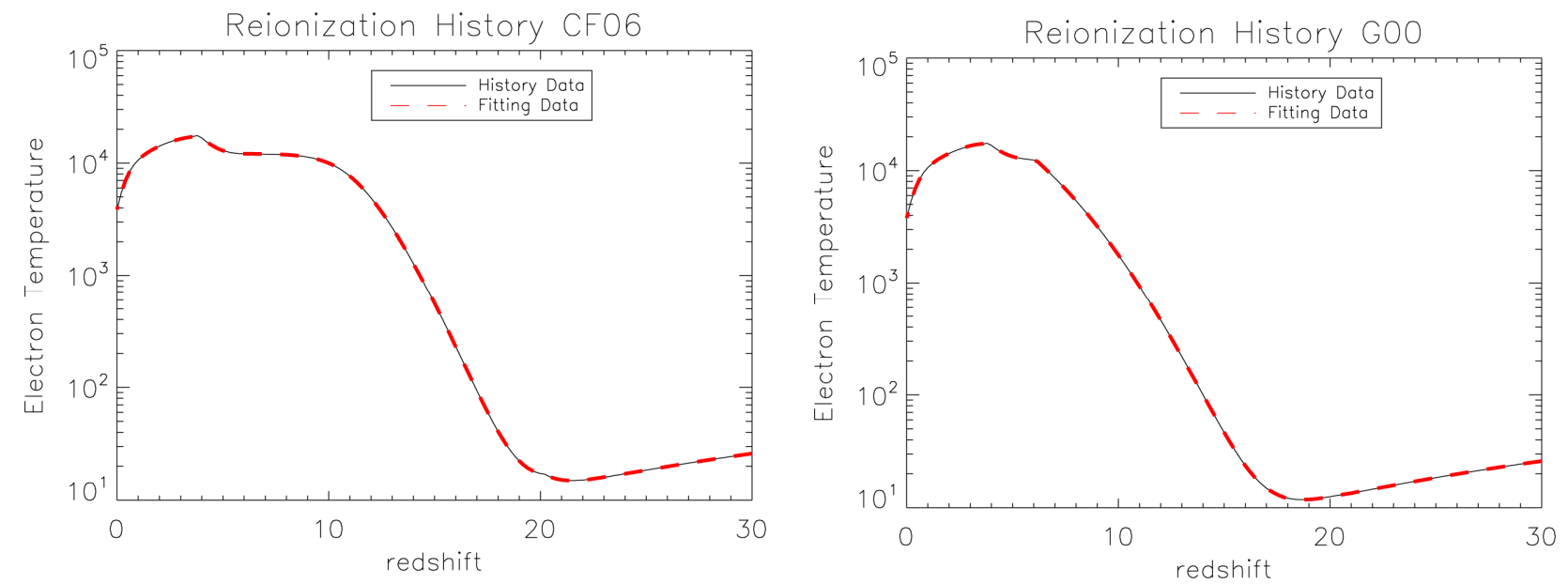

Figure 2. Electron temperature: comparison between the tabulated data (solid black line) and the fit (dashed red line) for the suppression (left panel) and filtering model (right panel).
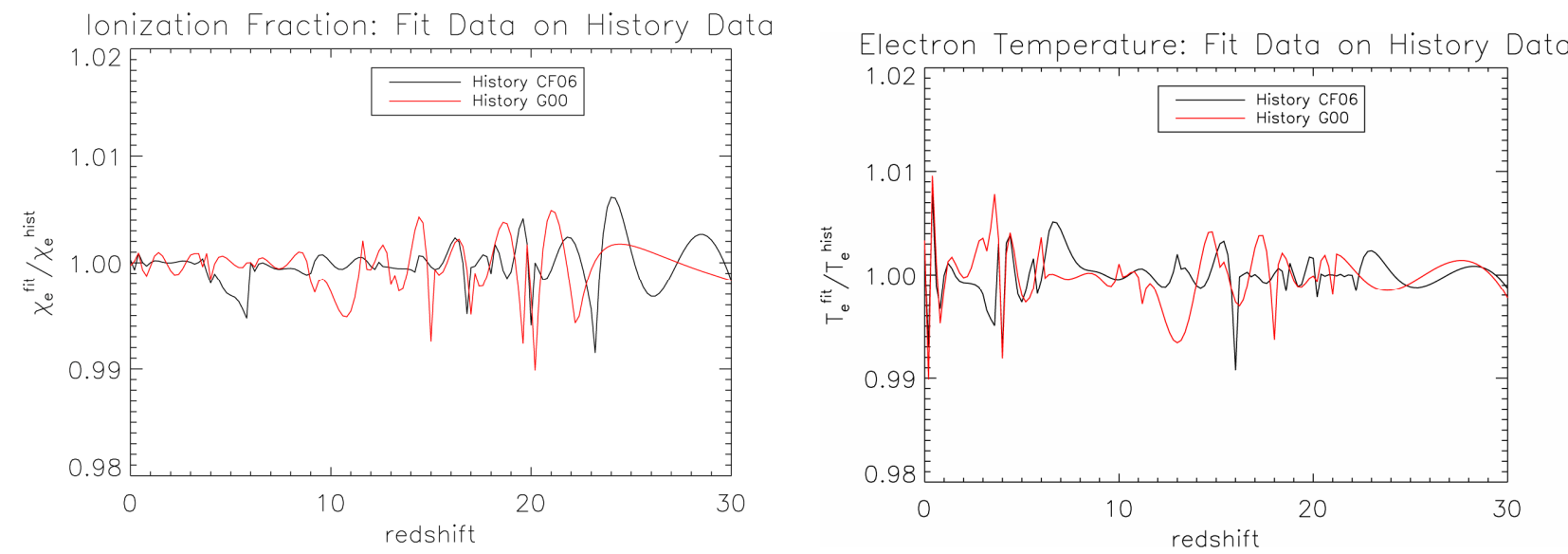

Figure 3. Ionization fraction: ratios between the fit functions and the tabulated data for the ionization fraction (left panel) and electron temperature (right panel) as function of redshift for the two different reionization histories.

picture. These models are based on the assumption that extra ionizing sources can be described by the efficiency of the ionizing photons production coefficient $\varepsilon_{i}$ which characterizes the ionizing photon production rate:

$$
\frac{\mathrm{d} n_{i}}{\mathrm{~d} t}=\varepsilon_{i}(z) n_{b}(z) H(z),
$$


where $n_{b}$ is the baryon density and $H(z)$ is the Hubble parameter.

The late reionization history has been modeled as:

$$
\begin{aligned}
\varepsilon_{i}(z)= & \varepsilon_{0} \exp \left[-\frac{\left(z-z_{r e}\right)^{2}}{\Delta z^{2}}\right] \\
& +\varepsilon_{1}(1+z)^{-m} \Theta\left(z_{r e}-z\right) .
\end{aligned}
$$

The two terms in the right hand of the equation describe the two ionizing epochs, with $\varepsilon_{0}, \varepsilon_{1}$ and $m$ free parameters of the model, $\Delta z$ the width of the first reionization epoch, $\Theta$ the step function. The first peak corresponds to a reionization fraction that decreases considerably at $z>z_{r e}$, while the second term characterizes a reionization fraction which is monotonic increasing with increasing time (or decreasing redshift).

For the early reionization history, the authors adopted a representations in which the efficiency coefficient has a Gaussian parametric form:

$$
\varepsilon_{i}(z)=\xi \exp \left[-\frac{\left(z-z_{r e}\right)^{2}}{\Delta z^{2}}\right]
$$

again, $\xi, Z_{r e}$ and $\Delta z$ are free parameters (clearly different from those of the previous scenario).

Suitable choices of these parameters allow us to model different reionization scenarios, and with an accurate balance of their values it is possible to obtain the desired value of the Thomson optical depth. In principle, one can derive the same value of $\tau$ with different combinations of these parameters, but the corresponding reionization histories will be different. As result, the predictions for the CMB APS will be also different. In order to present results spanning a wider range of possibilities and also to show the code versatility in ingesting parameter values implying larger displacements in CMB APS predictions, we exploited models not necessarily constrained by current data. Assuming (in both astrophysical and phenomenological models) the same cosmological parameters defined in the previous section, we selected sets of reionization parameters able to approximately reproduce the optical depth found for the suppression model, an astrophysical prescription (among those analyzed in this work) in agreement with current constraints on $\tau$, in order to focus on differences in APS even for models characterized by the same Thomson optical depth ${ }^{5}$.

In particular, the late history was modeled by this set of parameters:

\footnotetext{
${ }^{5}$ Clearly, one could search for combinations of all relevant parameters (i.e. related to the reionization model and to the underlying cosmological model) that match current observational data, but this is not the scope of the present work.
}

$$
\left\{\begin{array}{l}
\varepsilon_{0}=1.3 \times 10^{3} \\
z_{r e}=10 \\
\beta=\frac{\varepsilon_{1}}{10^{9}}=5.3 \times 10^{-6} \quad \Rightarrow \tau_{L}=\tau_{C F 06}=0.1017 \\
m=11.95 \\
\Delta z=0.025 z_{r e}
\end{array}\right.
$$

Concerning the early scenario, since it is unable (alone) to contribute to the actual optical depth having a substantial high reionization redshift, we exploited its combination with the filtering model, such as the global optical depth is exactly the same that figures out from the suppression model, finding the following set of values:

$$
\left\{\begin{array}{l}
\xi=2.315 \\
z_{r e}=500 \\
\Delta z=0.025 z_{r e}
\end{array} \Rightarrow \tau_{E}^{\text {tot }}=\tau_{E}+\tau_{G 00}=0.1017 .\right.
$$

In the case of early history, in order to satisfy the above condition on $\tau$ (for the considered choice of $\Delta z$ ) we investigated the dependence of the free variable $\xi$ on the reionization redshift, $z_{r e}$, in view of the broaden range of values that this utmost parameter can explore in comparison to a standard reionization model. As shown in Figure 4, in spite of an initial linear dependence at relatively low redshift, we observe a semi-parabolic behavior at higher redshifts.

The ionization fraction can be evaluated from the balance between the recombination and ionization processes:

$$
\frac{\mathrm{d} x_{e}}{\mathrm{~d} t}=-\alpha_{\text {rec }}(T) n_{b} x_{e}^{2}+\varepsilon_{i}(z)\left(1-x_{e}\right) H(z)
$$

where $\alpha_{\text {rec }} \sim 4 \times 10^{-13}\left(T / 10^{4} \mathrm{~K}\right)^{-0.6} \mathrm{~s}^{-1} \cdot \mathrm{cm}^{-3}$ is the recombination coefficient.

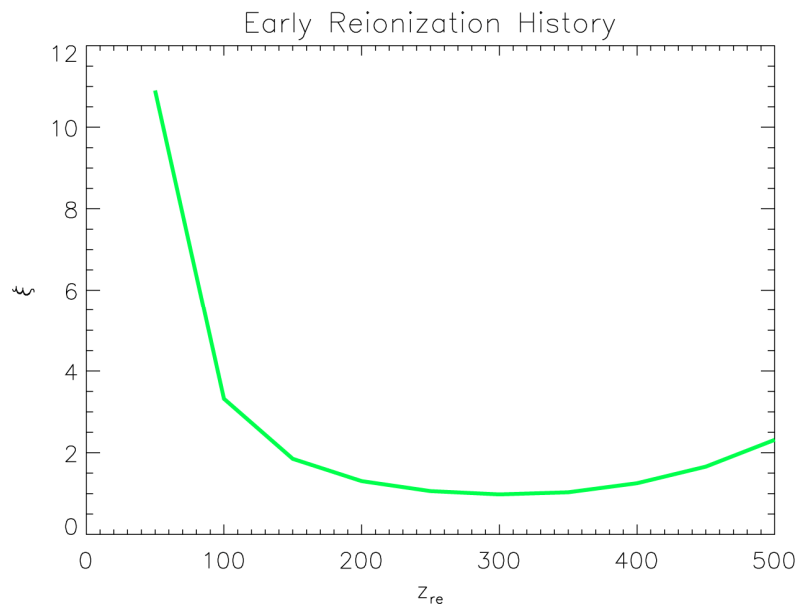

Figure 4. Dependence of the free parameter $\xi$ on the reionization redshift of the early history in order to have the adopted value of $\tau$. 
Assuming a curvature term $\Omega_{k}=0$, and so $\Omega_{\Lambda}=1-\Omega_{m}$ the Hubble parameter is approximated by:

$$
H(z)=H_{0} \sqrt{\Omega_{m}(1+z)^{3}+\Omega_{\Lambda}} .
$$

In Figure 5 we display the time evolution of the ionization fraction for all the models considered in this work. In particular, the coupled early and filtering model is plotted for three different cases of the reionization redshift. Note that, in order to have a constant optical depth, we varied the parameter $\xi$ assuming values $\xi=(2.315,1.031,1.309)$ when $z_{r e}=(500,350,200)$ respectively, giving a decreasing in the peak of the high redshift region of $x_{e}$, not linear with the reionization redshift. The reason can be found analyzing the redshift dependence of the recombination and ionization coefficients that are, respectively:

$$
C_{r e c} \propto n_{b} \propto z^{3},
$$

and

$$
C_{\text {ion }} \propto \varepsilon_{i}\left(1-x_{e}\right) H(z) \propto \xi z^{3 / 2},
$$

so that the higher is $z_{r e}$ the lower is $x_{e}$ (see Table $\mathbf{1}$ for details).

In these prescriptions the ionization fraction and electron temperature are provided in an analytical form and connected through the dependence on the temperature of the recombination coefficient $\alpha_{\text {rec }}$, that enters in the time evolution equation of the ionization fraction. Since

Table 1. Redshift dependence of recombination and ionization coefficients for decreasing reionization redshift and adjusted $\xi$ model parameter when joining the early reionization history with the filtering model.

\begin{tabular}{cccc}
\hline$\xi$ & $Z_{\text {re }}$ & $C_{\text {rec }}$ & $C_{\text {ion }}$ \\
\hline 2.315 & 500 & $1.25 \times 10^{8}$ & $2.59 \times 10^{4}$ \\
1.031 & 350 & $4.29 \times 10^{7}$ & $6.75 \times 10^{3}$ \\
1.309 & 200 & $8.00 \times 10^{6}$ & $3.70 \times 10^{3}$ \\
\hline
\end{tabular}

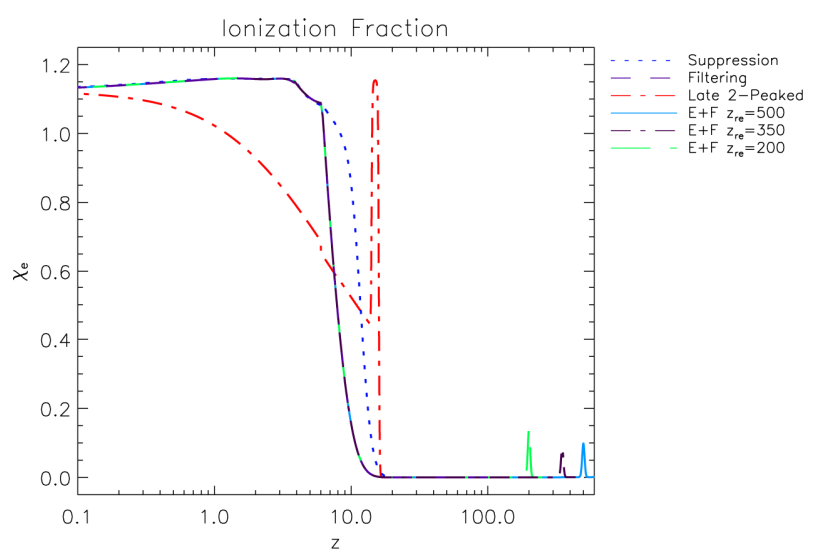

Figure 5. Reionization fraction: comparison between models. the relatively weak dependence, $\alpha_{\text {rec }} \propto T^{-0.6}$, the details of the assumptions on the matter temperature are not particularly critical, although, in principle, for the active phases out of equilibrium, they could play a non negligible role. For the active phase, we assume here, respectively, a temperature profile mimicking the model by Cen in the case of late processes and a temperature profile given by the same Gaussian parametric form as in Equation (3) but with a peak temperature, $T_{p}$, as free parameter instead of $\xi$ in the case of early processes, as adopted by Burigana and collaborators in 2004. We use here $T_{p}=6 \times 10^{4} \mathrm{~K}$. When the ionizing photons production is negligible and no longer affects the ionization history we assume the minimal ionization fraction usually derived in the absence of source terms, thus assuring the continuity with the quiescent phases in the evolution of the plasma properties.

\section{Comoving Fractions of Injected Photon Number and Energy Density}

For many mechanisms of cosmological reionization, the underlying physical process is usually characterized in terms of an additional source of ionizing photons injected in the plasma. We link here the parameters of the considered phenomenological histories to the comoving fractions of injected photon number density and energy density. Defining the usual CMB photon number density $n_{0} \simeq 399\left(T_{0} / 2.7 \mathrm{~K}\right)^{3}$, the comoving fractions of photon number density injected in the redshift range $z_{i} \geq z \geq z_{f}$ is given by:

$$
\frac{\Delta n}{n}=\int_{z_{f}}^{z_{i}} \frac{\varepsilon_{i}(z) n_{b}(z)}{n_{0}(1+z)^{4}} \mathrm{~d} z .
$$

The second term, $\varepsilon_{1}(1+z)^{-m} \Theta\left(z_{r e}-z\right)$, appearing in the late reionization history can be easily integrated:

$$
\frac{\Delta n}{n}=\int_{0}^{z_{r e}} \frac{\varepsilon_{1} n_{b 0}}{n_{0}(1+z)^{1+m}} \mathrm{~d} z=\frac{\varepsilon_{1} n_{b 0}}{n_{0} m}\left[1-\left(1+z_{r e}\right)^{-m}\right],
$$

where $n_{b 0}=1.12 \times 10^{-5} \Omega_{b} h^{2}$ is the current baryon number density.

For the Gaussian term defining the early model and appearing as first term in the late model, we find the following suitable approximation:

$$
\begin{aligned}
& \frac{\Delta n}{n}=\int_{z_{f}}^{z_{i}} \frac{\text { const } \cdot n_{b 0}}{n_{0}(1+z)} \exp \left[-\frac{\left(z-z_{r e}\right)^{2}}{\Delta z^{2}}\right] \mathrm{dz} \\
& \simeq \frac{\text { const } \cdot n_{b 0}}{n_{0}} \frac{\Delta z}{1+z_{r e}} \sqrt{\pi},
\end{aligned}
$$

where "const" is the early parameter $\xi$ or the late parameter $\varepsilon_{0}$, respectively, and the assumption $z_{f} \rightarrow-\infty$ and $Z_{i} \rightarrow \infty$ for the integration limits is made, a good approximation to this aim for a peaked Gaussian shape. 
The comoving fraction of injected photon energy density depends on the energy distribution function of ionizing photons. Given the comoving fraction of injected photon number density and assuming the mean ionizing photon energy, $\bar{E}_{\gamma}^{i o n}$, here assumed $\simeq 10 \mathrm{eV}$ for numerical estimates, one can compute of the comoving fraction of injected photon energy density, $\Delta \varepsilon / \varepsilon$. In the case of the early history and for the Gaussian term of the late history we have:

$$
\frac{\Delta \varepsilon}{\varepsilon} \simeq 1.59 \times 10^{4} \frac{\Delta n}{n} \frac{\bar{E}_{\gamma}^{\text {ion }}}{10 \mathrm{eV}}\left(\frac{T_{0}}{2.7}\right)^{-1}(1+\mathrm{z})^{-1}
$$

where for numerical estimates we can assume $(1+z) \simeq\left(1+z_{r e}\right)_{\text {early/late }}$. For the second term appearing in the late reionization history, after a simple integration we have:

$$
\begin{aligned}
& \frac{\Delta \varepsilon}{\varepsilon} \simeq 1.60 \times 10^{-11} \frac{\bar{E}_{\gamma}^{\text {ion }}}{10 \mathrm{eV}} \frac{\varepsilon_{1} n_{b 0}}{\varepsilon_{0}(m+1)} \\
& \cdot\left[1-\left(1+z_{r e}\right)^{-(m+1)}\right],
\end{aligned}
$$

where $\varepsilon_{0}=a T_{0}^{4}$ is the current $\mathrm{CMB}$ photon energy density.

In general, we used the D01AJF routine of the NAG Libraries for an accurate numerical cross-check of previous analytical formulas and approximations. For the adopted parameters we found an agreement better than $\simeq 0.03 \%$.

\section{Code Implementation}

We modified CAMB [57], the cosmological Boltzmann code (see also [58]) for computing the angular power spectrum of the anisotropies of the $\mathrm{CMB}$, in order to introduce the ionization fractions evaluated according to the astrophysical and phenomenological reionization models described in previous sections, alternative to the reionization treatment originally implemented in CAMB. Of course, the methods described here can be used as guidelines for the implementation of any other astrophysical reionization model (see Appendix B for further details).

As a significant step forward with respect to previous analyses, the emphasis of this work is posed to the extension to a first detailed characterization of the polarization B-mode APS.

By implementing the source file reionization. $f 90$, that defines the Reionization module, we are able to parametrize the desired reionization history and to supply the corresponding ionization fraction as function of redshift [59].

Particular care must be taken to the normalization of the quantities occurring in the history definition, such as the ionization fraction. In CAMB the reionization frac- tion is referred to the hydrogen, so, when allowing for helium reionization, the global ionization fraction in the case of complete ionization can be greater than one, following the relation:

$$
\chi_{e}^{\text {full }}=1+\frac{n_{H e}}{n_{H}} .
$$

Instead, in the two astrophysical reionization models, the global ionization fraction corresponding to the case of complete ionization is $\chi_{e}^{\text {full,CF06 }}=1.12721$ for the suppression model, and $\chi_{e}^{\text {full,G00 }}=1.12480$ for the filtering model.

To evaluate the total fraction in the case of the phenomenological histories we have normalized it with CAMB implementation, such as:

$$
x_{e}^{\mathrm{CAMB}}=\frac{n_{e}}{n_{H}}=x_{e}^{L, E} \frac{1+f_{H}}{2 f_{H}},
$$

where $f_{H}$ is the fraction of baryonic mass in hydrogen. Assuming hydrogen abundance $f_{H}=0.76$, the global ionization fraction in the case of complete ionization for both histories is $f_{L, E}=1.157895$.

Furthermore, it is possible to fix in CAMB the Thomson optical depth parameter, and let the code estimates the reionization redshift, or to choose the desired reionization redshift, and obtaining from dedicated function the $\tau$ evaluation. While for the considered astrophysical models $\tau$ is known, we implemented a modified version of this function to derive $\tau$ according to the considered phenomenological model.

\section{Astrophysical Models and Standard CAMB}

In the framework of our adapted version of CAMB code it can be useful to analyze an important differences between the various models we described. The crucial characteristic resides in the interplay between different physical processes at various epochs which contribute to reionize the cosmological plasma. Every mixture of these processes can lead to a distinctive scenario, as displayed in Figure 5.

Since the standard CAMB traces a reionization that follows the "single peak" evolution scheme, it can be interesting to compare it with the suppression and filtering models, investigating on their differences in the APS and, in particular, in the sequence of acoustic peaks, at various multipoles (see Figures 6 and 7).

To this aim we derived the temperature (TT), polarization (EE and BB), and cross-correlation (TE) APS of $\mathrm{CMB}$ anisotropies for the two astrophysical reionization histories, CF06 and G00, generically denoted as "models" in titles and the legends, and for the original version of the code, denoted as "CAMB CF" or "CAMB G", assuming, respectively, an optical depth corresponding to 

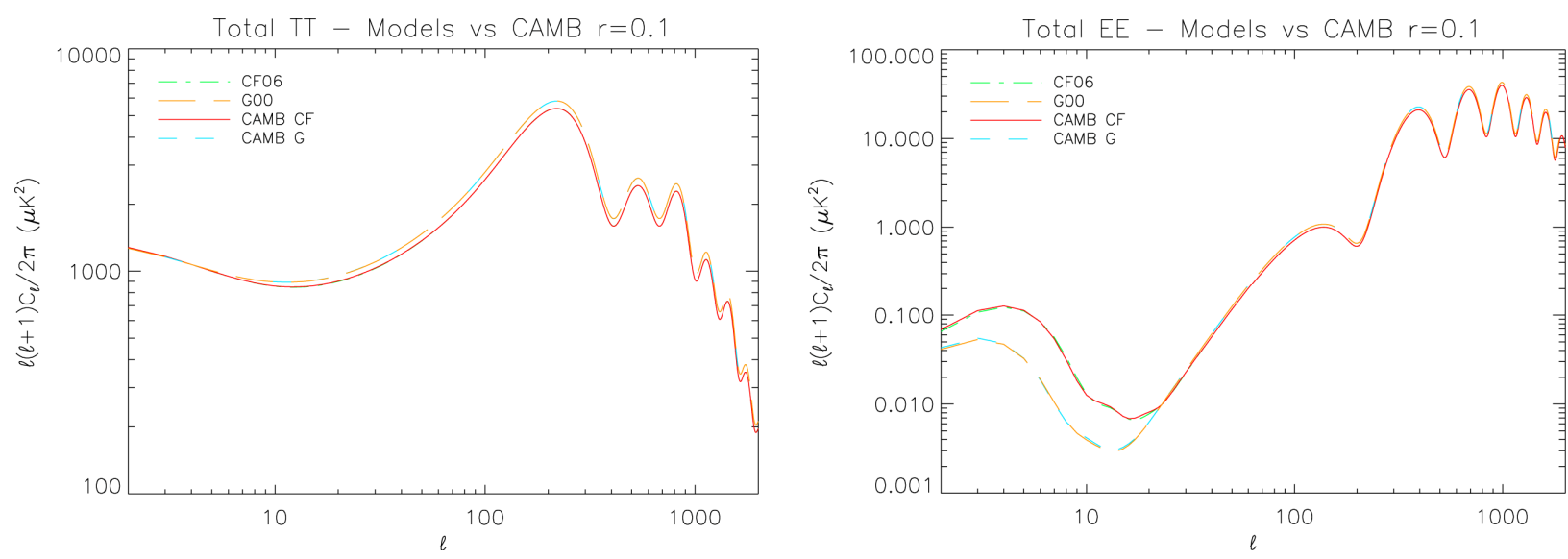

Figure 6. Comparison between the models and CAMB in temperature (left panel) and E-mode polarization (right panel) APS.
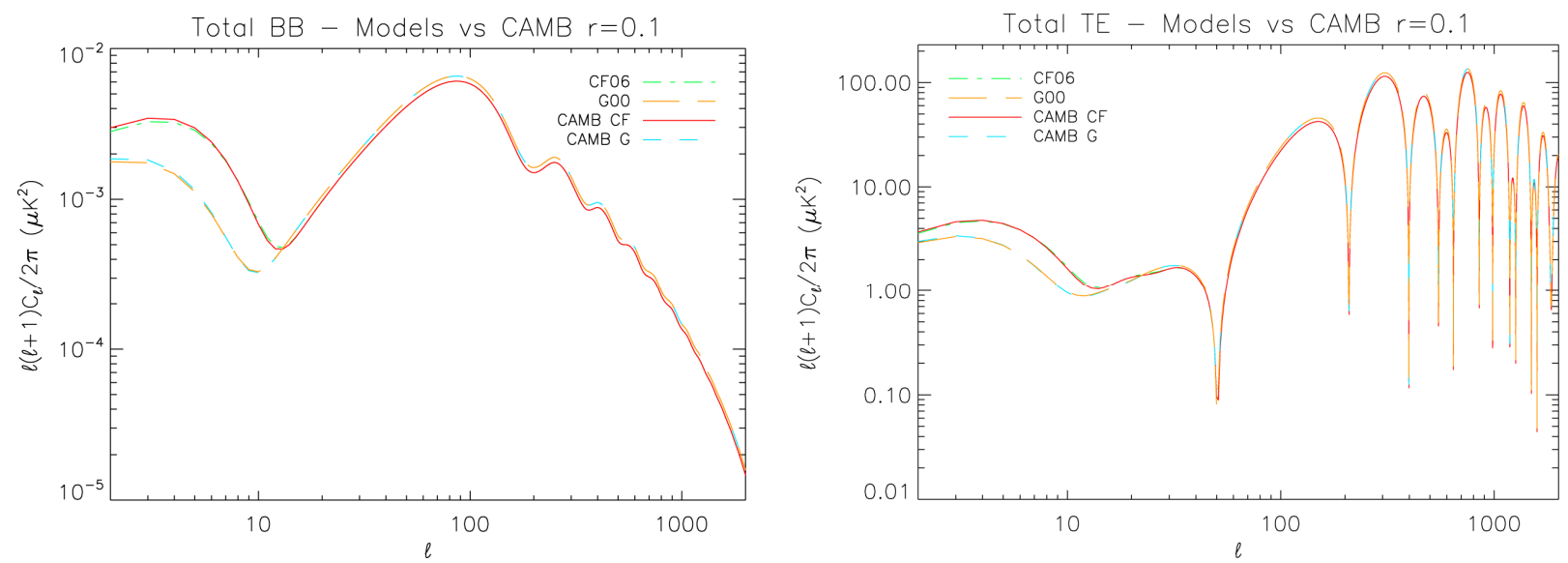

Figure 7. Comparison between the models and CAMB in B-mode polarization (left panel) and temperature-polarization cross-correlation (right panel) APS.

the value given by the theoretical model to which we are comparing to. Note also that in the right panel of Figure 7 we display the module of the cross-correlation APS.

For simplicity, we neglect lensing in this case, and the total $C_{\ell}$ plotted represents the sum of scalar and tensor contributions. The tensor to scalar ratio of primordial perturbation, $r$, is assumed here equal to 0.1 .

It is also very useful to analyse the relative difference between results obtained with the astrophysical models and the original CAMB, as shown in Figures 8 and $\mathbf{9}$, defined by the relation:

$$
\frac{C_{\ell}^{\text {Model }}-C_{\ell}^{\text {CAMB }}}{1 / 2\left(C_{\ell}^{\text {Model }}+C_{\ell}^{\text {CAMB }}\right)} .
$$

As before, note that in Figure 9 we display the module of the difference of the cross-correlation APS.

The relative differences found in the case of the suppression model are larger than in the case of the filtering prescription, mainly in the polarization and cross-correlation patterns of the APS. In addition, the difference is remarkable at low multipoles, in particular at $\ell<$ few tens, i.e. at intermediate and large angular scales, as intuitively expected since we are considering relatively late reionization processes.

\section{Sensitivity of CMB Measurements and Foreground Emission}

CMB experimental data are affected by uncertainties due to instrumental noise (crucial at high multipoles, $\ell$, i.e. small angular scales), cosmic and sampling variance (crucial at low $\ell$, i.e. large angular scales) and from systematic effects. Also, they are contaminated by a significant level of foreground emission of both Galactic and extragalactic origin. In polarization, the most critical Galactic foregrounds are certainly synchrotron and thermal dust emission, while free-free emission is negligible in polarization and other components, like spinning dust and haze, are still poorly known, particularly in polarization. Synchrotron emission is the dominant Galactic foreground signal at low frequencies, up to $\sim 60 \mathrm{GHz}$, where dust emission starts to dominate. External galaxies 

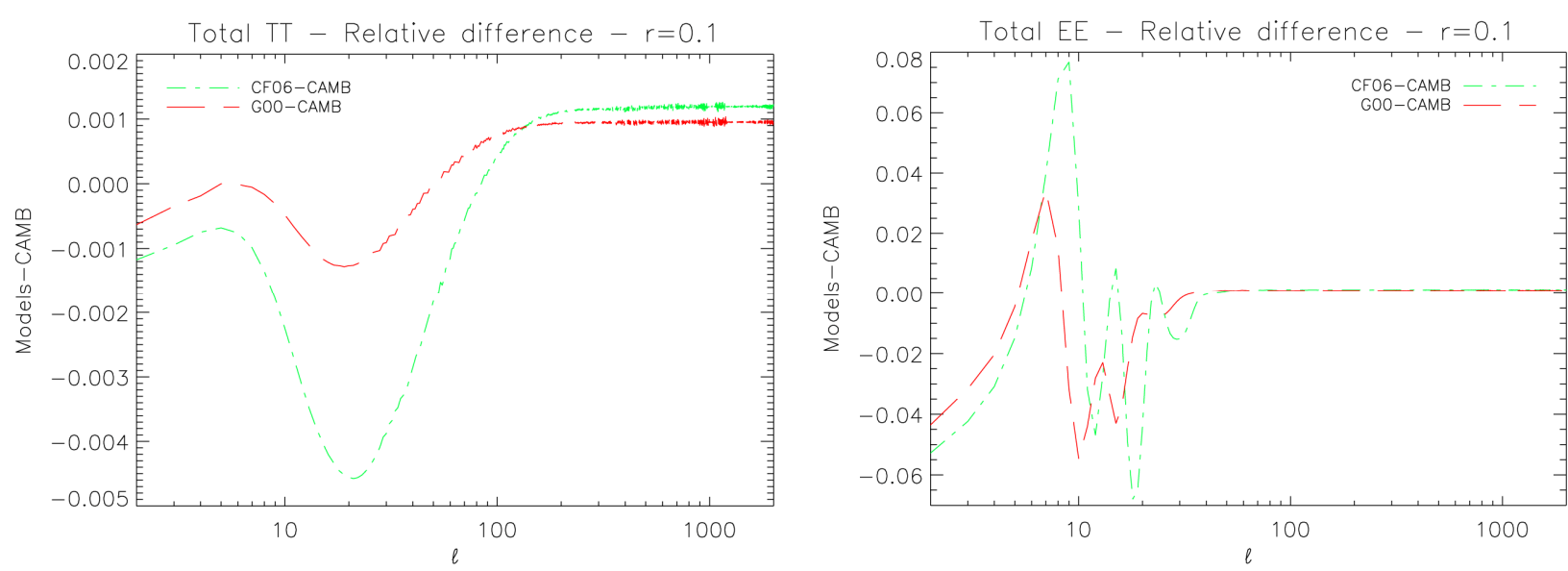

Figure 8. TT (left panel) and EE (right panel) APS: relative differences between the models and CAMB.
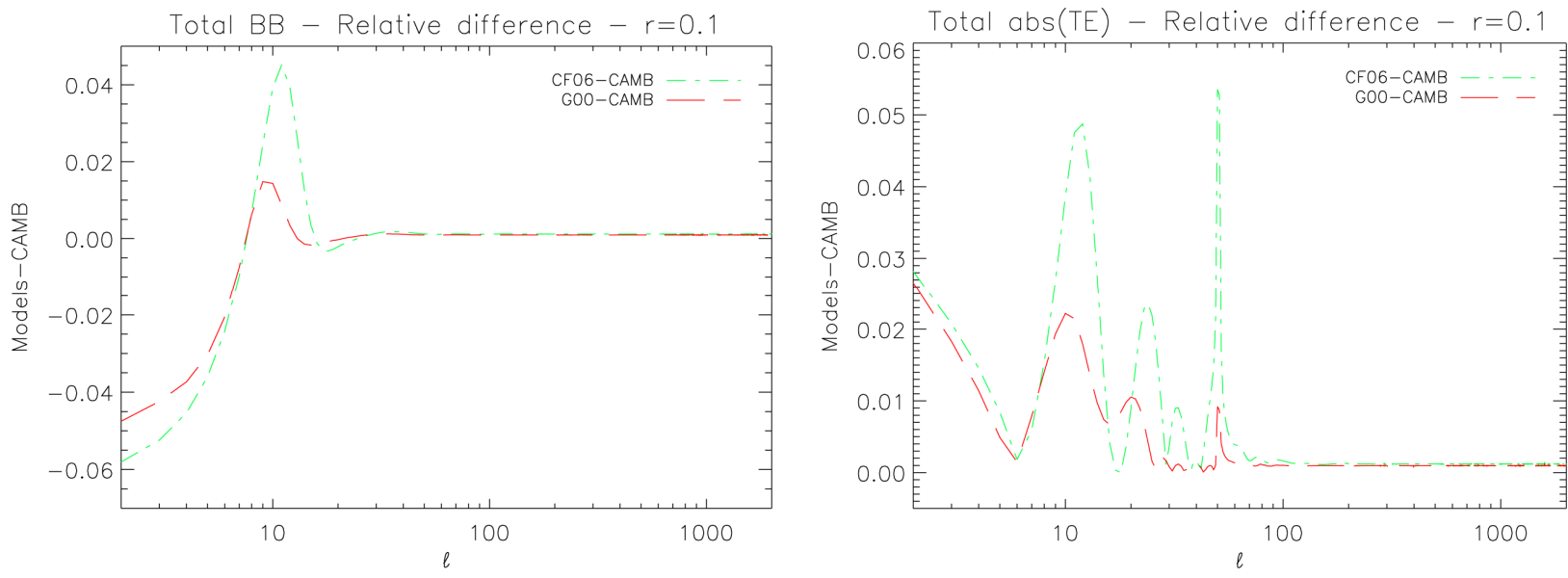

Figure 9. BB (left panel) and TE (right panel) APS: relative differences between the models and CAMB.

are critical only at high $\ell$, and radiogalaxies are likely the most crucial in polarization up frequencies $\sim 200 \mathrm{GHz}$, most suitable for CMB anisotropy experiments.

In this section we provide simple recipes aimed at evaluating the levels of sensitivity of on-going and future CMB space experiments and the kind of contamination expected from foregrounds, and to make us able discuss in the next section on the possibility to distinguish between different reionization scenarios in the framework of current and future experiments, focussing in particular on the pure polarization $\mathrm{E}$ and B-modes.

\subsection{Sensitivity Measurements}

The uncertainty on the angular power spectrum is given by the combination of three components, CV, SV and Instrumental Noise (N) [60]:

$$
\frac{\delta C_{\ell}}{C_{\ell}}=\sqrt{\frac{2}{f_{\text {sky }}(2 \ell+1)}}\left(1+\frac{A \sigma^{2}}{N C_{\ell} W_{\ell}}\right) .
$$

Here $f_{\text {sky }}$ is the sky coverage, $A$ is the surveyed area, $\sigma$ is the instrumental rms noise per pixel, $N$ is the total pixel number, $W_{\ell}$ is the beam window function that, in the case of a Gaussian symmetric beam, is:

$$
W_{\ell}=\exp \left(-\ell(\ell+1) \sigma_{B}^{2}\right)
$$

being $\sigma_{B}=F W H M / \sqrt{8 \ln 2}$ the beamwidth which defines the experiment angular resolution.

For $f_{\text {sky }}=1$ the first term in parenthesis defines the cosmic variance, an intrinsic limit on the accuracy at which the angular power spectrum of a certain cosmological model defined by a suitable set of parameters can be measured with the CMB. It typically dominates the uncertainty on the APS at low $\ell$ because of the small, $2 \ell+1$, number of modes $m$ for each $\ell$. The second term in parenthesis characterizes the instrumental noise, that never vanishes in the case of real experiments. Note also the coupling between experiment sensitivity and resolution, the former defining the low $\ell$ experimental uncertainty, namely for $W_{\ell}$ close to unit, the latter determining the exponential loss in sensitivity at angular scales comparable with the beamwidth. 
In order to provide concrete estimates of these quantities, we consider Planck LFI and HFI channels at $v=(70,100,143,217) \mathrm{GHz}$, and COrE channels at $v=(75,105,135,165,195,225) \mathrm{GHz}$, i.e. at the frequencies particularly suitable for CMB analyses because of the combination of good experimental sensitivity and resolution, and of relatively low foreground contamination. We adopt here the sensitivities and resolutions summarized in the COrE white paper ${ }^{6}$.

For each of the two projects we computed an overall sensitivity value, weighted over the channels, defined by

$$
\frac{1}{\sigma_{j, t o t}^{2}}=\sum_{i} \frac{1}{\sigma_{j, i}^{2}}
$$

where $j=T, P o l$ and $i$ states for the sensitivity of each frequency channel, listed in Tables 2 and 3. FWHM values of $13^{\prime}$ and $14^{\prime}$ are used to define the overall resolution respectively of Planck and COrE in the computation of the beam window function ${ }^{7}$.

Finally, to improve the signal to noise ratio in the APS sensitivity, especially at high multipoles, we will apply a multipole binning of $5 \%$ in temperature APS, $15 \%$ in TE cross-correlation and 30\% in polarization APS, both in $\mathrm{E}$ and $\mathrm{B}$-modes.

Table 2. Instrumental sensitivity of Planck experiment.

\begin{tabular}{cccc}
\hline$v(\mathrm{GHz})$ & $F W H M(\operatorname{arcmin})$ & $\sigma_{T}(\mu \mathrm{K}$ arcmin $)$ & $\sigma_{\text {Pol }}(\mu \mathrm{K}$ arcmin $)$ \\
\hline 70 & 13 & 211.2 & 298.7 \\
100 & 9.9 & 31.3 & 44.2 \\
143 & 7.2 & 20.1 & 33.3 \\
217 & 4.9 & 28.5 & 49.4 \\
\hline
\end{tabular}

Table 3. Instrumental sensitivity of COrE experiment.

\begin{tabular}{cccc}
\hline$v(\mathrm{GHz})$ & $F W H M(\operatorname{arcmin})$ & $\sigma_{T}(\mu \mathrm{K}$ arcmin $)$ & $\sigma_{\text {Pol }}(\mu \mathrm{K}$ arcmin $)$ \\
\hline 75 & 14 & 2.73 & 4.72 \\
105 & 10 & 2.68 & 4.63 \\
135 & 7.8 & 2.63 & 4.55 \\
165 & 6.4 & 2.67 & 4.61 \\
195 & 5.4 & 2.63 & 4.54 \\
225 & 4.7 & 2.64 & 4.57 \\
\hline
\end{tabular}

${ }^{6}$ The nominal sensitivity of Planck is slightly better than that adopted here thanks the slightly longer extension of the mission with both instruments, and the additional extension with the only LFI. Of course, the real sensitivity of the whole mission will have to include also the potential residuals of systematic effects.

${ }^{7}$ In fact, it is possible to smooth maps acquired at higher frequencies with smaller beamwidths to the resolution corresponding to the lowest frequency of each experiment.

\subsection{Parametrization of Residual Polarized Foreground Contamination}

The parametrization of the APS of Galactic thermal dust and synchrotron emission adopted in this work is taken from the results of WMAP 3-yrs [61] under the assumption that these contributions are uncorrelated ${ }^{8}$, and is expressed by:

$$
\frac{\ell(\ell+1)}{2 \pi} C_{\ell}^{\text {fore }}=\left(\mathcal{B}_{s}(v / 65)^{2 \beta_{s}}+\mathcal{B}_{d}(v / 65)^{2 \beta_{d}}\right) \ell^{m},
$$

where $s$ and $d$ stands for synchrotron and dust, and the frequency $v$ is expressed in GHz. The coefficients characterizing the E and B-modes polarization APS are slightly different, and are listed in Table 4.

In the next sections we will parametrize a potential residual from non perfect cleaning of CMB maps from Galactic foregrounds simply assuming that a certain fraction of the foreground signal at map level (or, equivalently, its square at power spectrum level) contaminates CMB maps. Of course, one can easily rescale the following results to any fraction of residual foreground contamination. The frequency of $70 \mathrm{GHz}$, i.e. the Planck channel where Galactic foreground is expected to be minimum at least at angular scales above $\sim$ one degree, will be adopted as reference.

For what concerns extragalactic source fluctuations $[62,63]$, we will adopt the recent (conservative) estimate of their Poissonian contribution to the APS [64] at 100 $\mathrm{GHz}^{9}$ assuming a detection threshold of $\simeq 0.1 \mathrm{Jy}$, together with a potential residual coming from an uncertainty in the subtraction of this contribution computed assuming a relative uncertainty of $\simeq 10 \%$ in the knowledge of their degree of polarization and in the determination of the source detection threshold, implying a reduction to $\simeq 30 \%$ of the original level [65]. Except at very high multipoles, their residual is likely significantly below that coming from Galactic foregrounds.

\section{Results}

In this section we present the angular power spectra

Table 4. Parametrization of $E$ and $B$ mode polarization power spectra of Galactic synchrotron and thermal dust emission.

\begin{tabular}{cccccccc}
\hline E-mode & $\mathcal{B}(\mu \mathrm{K})^{2}$ & $\beta$ & $m$ & B-mode & $\mathcal{B}(\mu \mathrm{K})^{2}$ & $\beta$ & $m$ \\
\hline sync & 0.36 & -3.0 & -0.6 & sync & 0.3 & -2.8 & -0.6 \\
dust & 1.00 & 1.5 & -0.6 & dust & 0.5 & 1.5 & -0.6 \\
\hline
\end{tabular}

${ }^{8} \mathrm{~A}$ more sophisticated treatment of the foreground power spectra takes into account the correlation among the various foreground components. ${ }^{9} \mathrm{We}$ adopt here a frequency slightly larger than that considered for Galactic foregrounds because at small angular scales, where point sources are more critical, the minimum of foreground contamination is likely shifted at higher frequencies. 
resulting from different prescriptions of the reionization process and compare them with the sensitivities of on-going and future space experiments including instrumental noise and fundamental statistical uncertainty.

Figures 10-13, show the temperature, polarization and cross-correlation APS up to multipoles $\ell=2000$ for a range of values of the so-called tensor-to-scalar ratio, the $r$ parameter (or $T / S$ ), which parametrizes the ratio between the amplitudes of primordial tensor and scalar perturbations. We also show the estimated cosmic and sampling variance, and instrumental noise contributions for the Planck (dotted lines) and COrE (dash-dotted lines) experiments, assuming a sky coverage of $80 \%$ and a multipole binning of $5 \%, 30 \%$ and $15 \%$ depending on the pattern.

As expected, the temperature APS does not exhibit a remarkable dependence on the $T / S$ parameter, as evi-
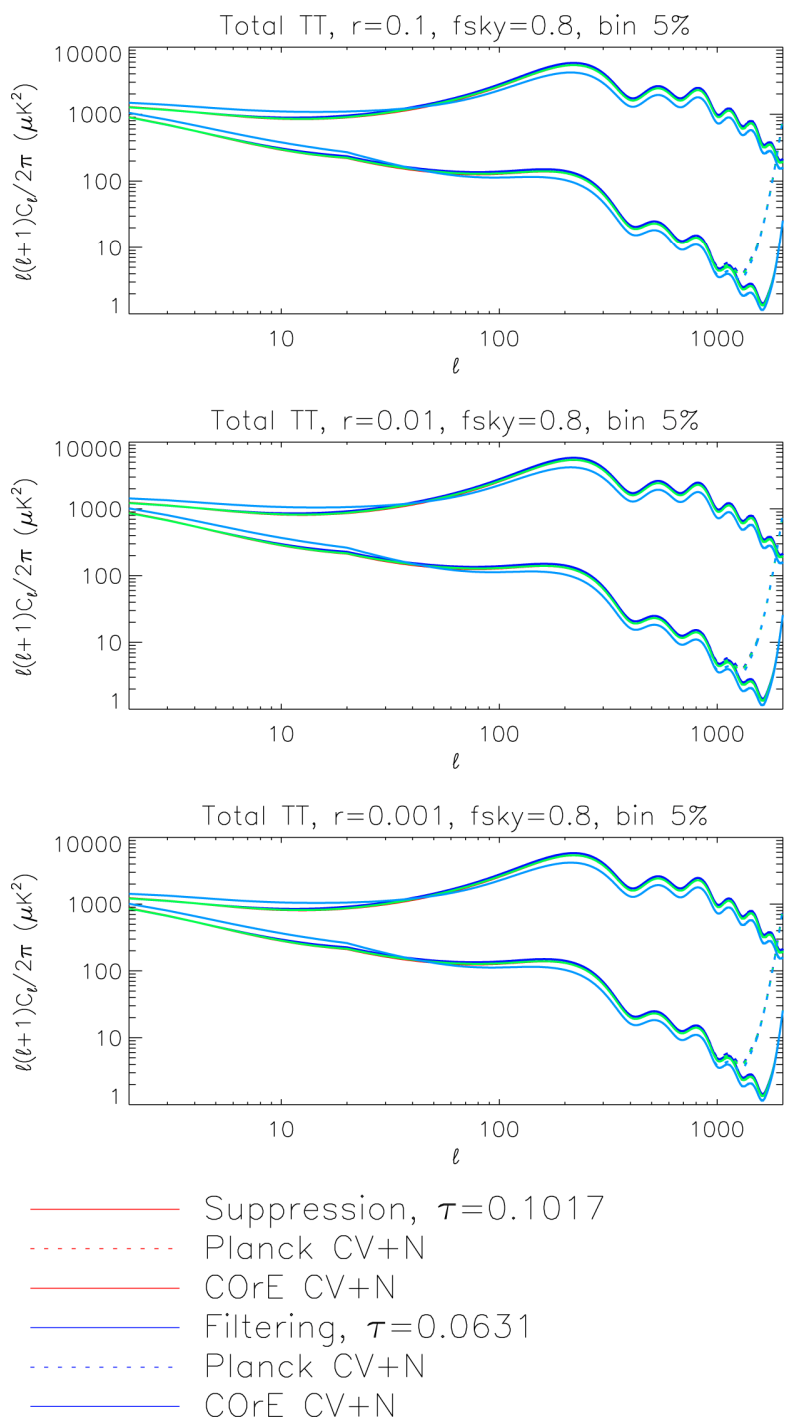

dent from the comparison between the panels in Figure 10. Comparing the considered reionization models, we observe a difference between their relative power at high and low multipoles: in particular, the early plus filtering curves show more power at low $\ell$ and less at high $\ell$. Note that this double peaked reionization history has a high redshift phase characterized by a remarkable ionization fraction.

The E-mode power spectrum is shown in Figure 11. Again, it is only weakly dependent on the $T / S$ parameter, but for early plus filtering model that is widely different from the other models up to the second acoustic peak. Note also that, even if suppression and late (double peaked) models have the same optical depth and cosmological parameters, their power spectra are significantly different in both shape and power. For the late model, the reionization bump is slightly shifted to higher multipoles
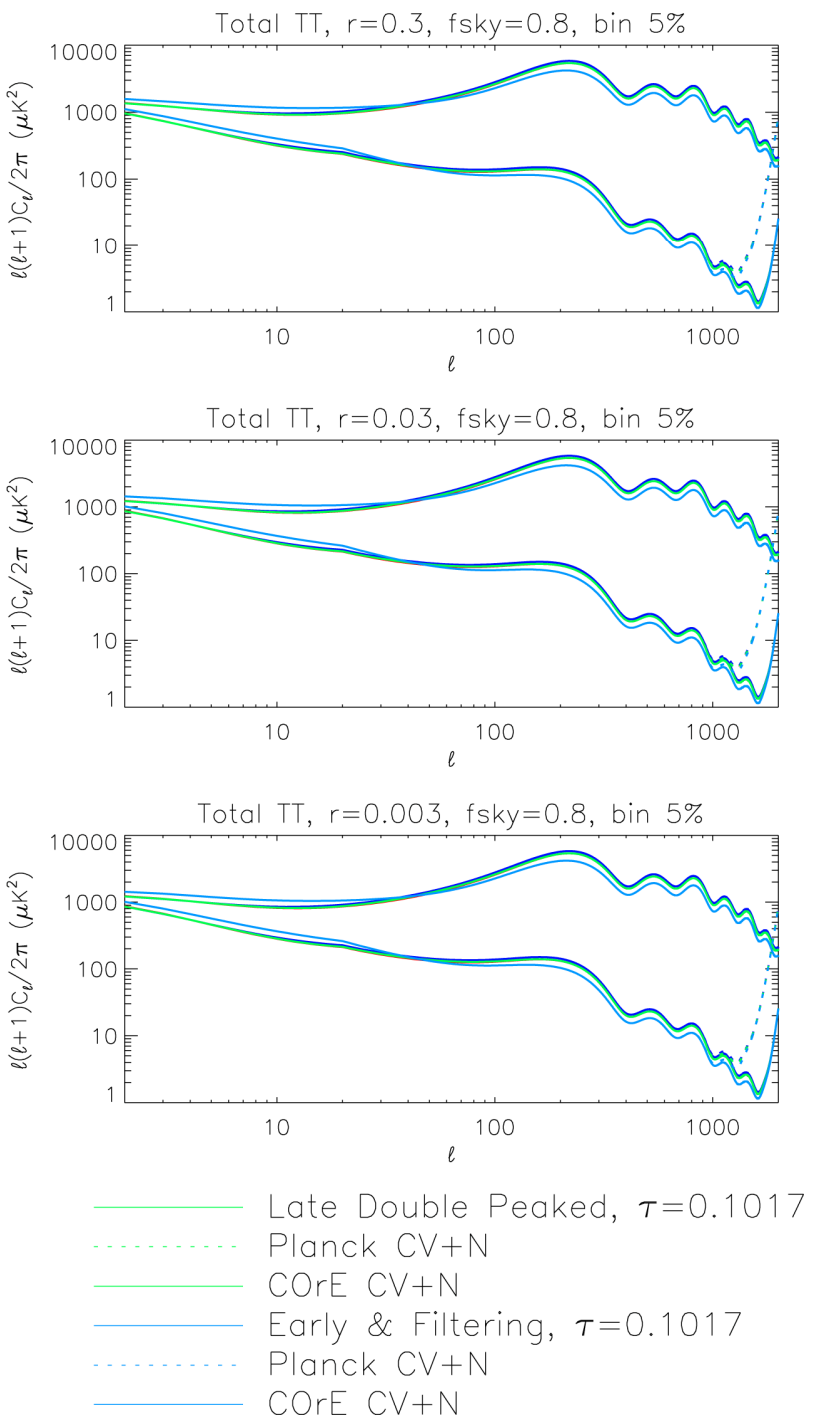

Figure 10. TT APS for the reionization histories: suppression, filtering, late double peaked, early plus filtering for different values of tensor to scalar ratio (see plot legend and text for details). 

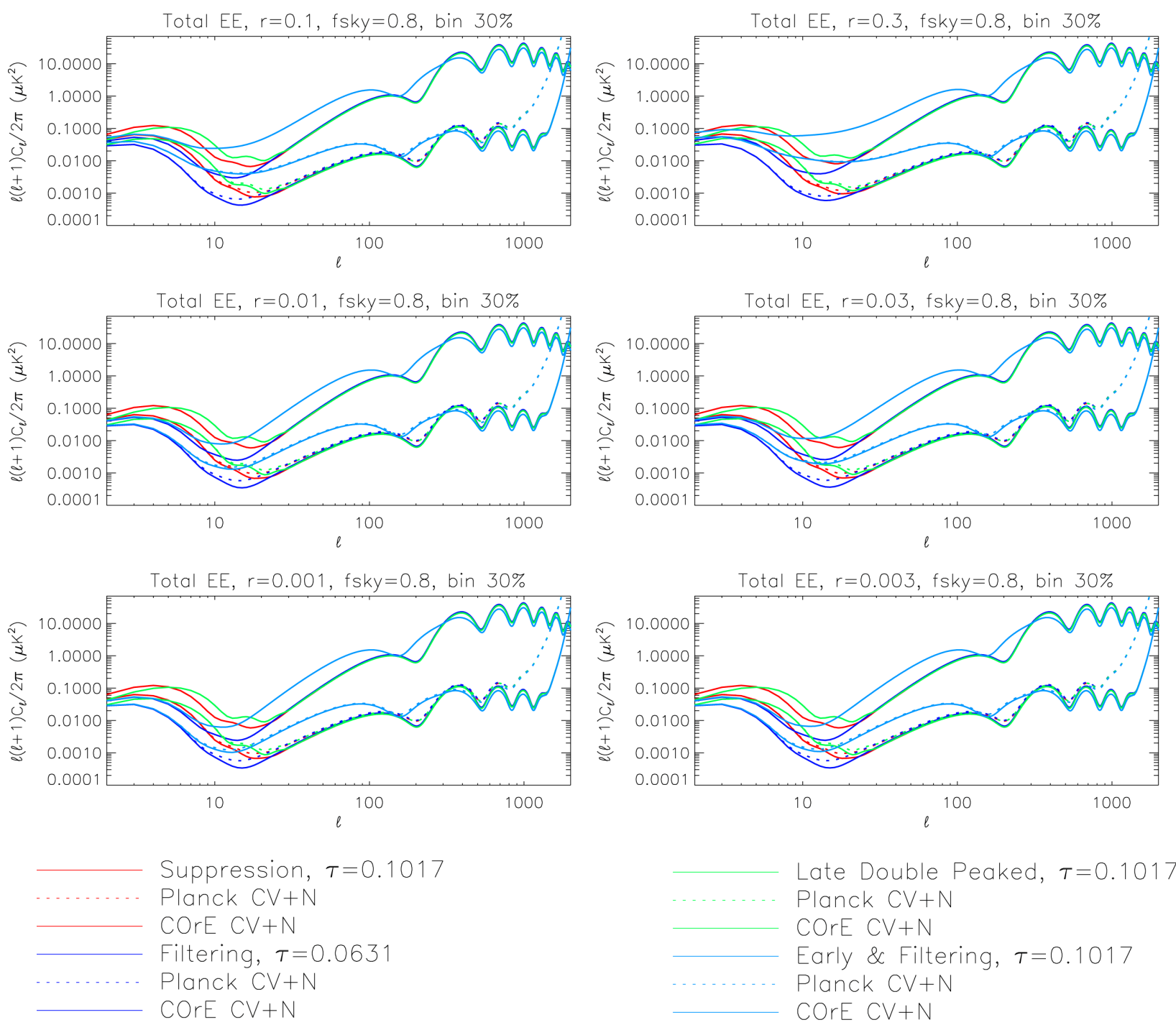

Figure 11. EE APS for the reionization histories: suppression, filtering, late double peaked, early plus filtering for different values of tensor to scalar ratio $r$ (see plot legend and text for details).

with respect to the other histories.

The B-mode power spectrum (see Figure 12) plotted here including also the lensing contribution, shows the expected linear dependence on $T / S$ at low multipoles, where the primordial signal dominates over the lensing contribution, which, on the contrary, determines the power at high $\ell$ almost independently of $r$ because of the relative weight of primordial and lensing signal. The tensor-to-scalar ratio parametrizes the B-mode anisotropies from inflation resulting on a strong impact on the shape of the observable acoustic peaks, in particular the first one, affected by reionization and flattened for decreasing values of $r$, and the second one, more related to the recombination epoch and centered around $\ell \simeq 70$. These two peaks inevitably plays a fundamental role in the study of the reionization history and consequently on the first structures formation in the universe. Actually, while tensors contribute to the E-mode polarization pattern as well as scalars, the primordial B-modes are generated only by tensor perturbations, so detecting them allow us to indirectly probe the stochastic field of primordial gravity waves (see e.g. [7,8]; see also [9-11]). Meanwhile, to firmly achieve this aim is required a resolution of at least $\simeq 1^{\circ}$ [65], maybe lower than that of Planck, but a significantly better sensitivity, as that foreseen for a next generation of experiments like COrE, depending on the tensor-to-scalar ratio, while a resolution of few arcminutes would greatly help the disentangle between primordial and lensing B-modes, being also crucial for the study of other crucial topics in cosmology (see e.g. [36]).

Typically, the reionization bump is stronger for the suppression and the late (double peaked) models, weaker for the filtering and the early plus filtering histories. 
Total BB \& Lensing, $r=0.1$, fsky $=0.8$, bin $30 \%$

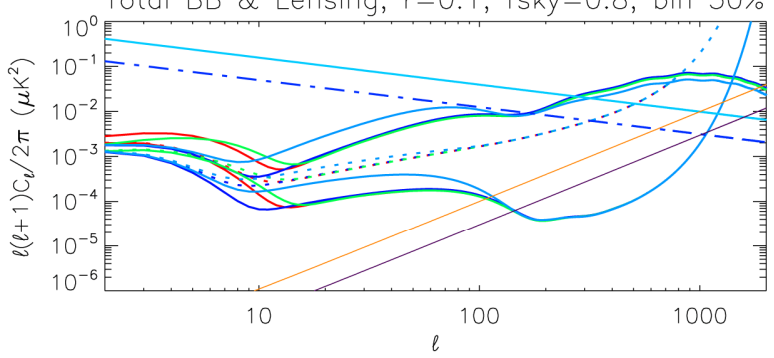

Total BB \& Lensing, $r=0.01$, fsky $=0.8$, bin $30 \%$

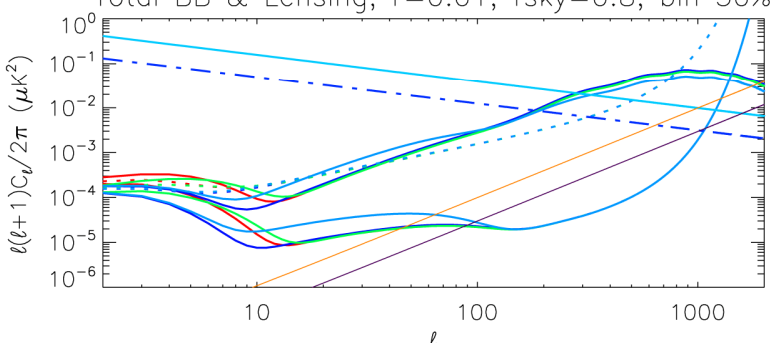

Total BB \& Lensing, $r=0.001, f s k y=0.8$, bin $30 \%$

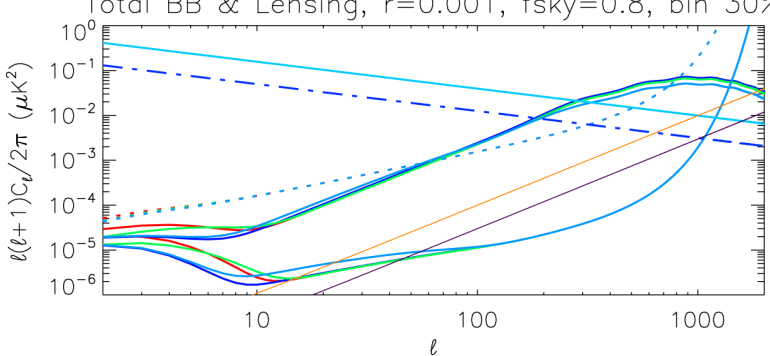

Suppression, $\tau=0.1017$

Planck $\mathrm{CV}+\mathrm{N}$

COrE $\mathrm{CV}+\mathrm{N}$

Filtering, $\tau=0.0631$

Planck $\mathrm{CV}+\mathrm{N}$

COrE CV $+\mathrm{N}$

Synchrotron, $\nu_{\mathrm{cmb}}=70 \mathrm{GHz}$

Radiosources

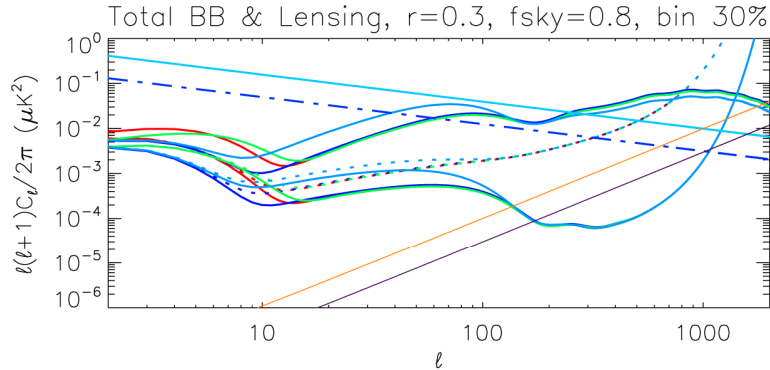

Total BB \& Lensing, $r=0.03$, fsky $=0.8$, bin $30 \%$

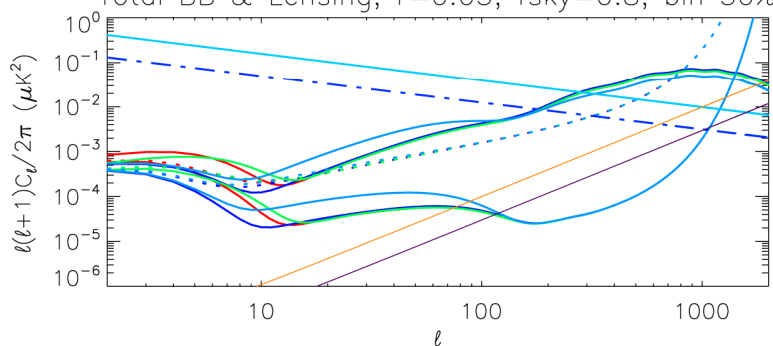

Total BB \& Lensing, $r=0.003$, fsky $=0.8$, bin $30 \%$

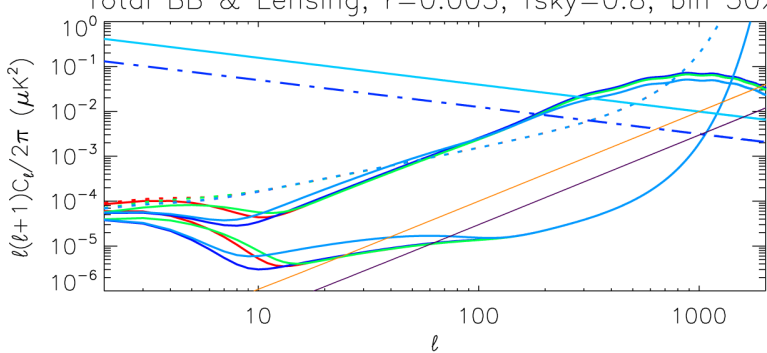

Late Double Peaked, $\tau=0.1017$

Planck $\mathrm{CV}+\mathrm{N}$

COrE CV+N

Early \& Filtering, $\tau=0.1017$

Planck $\mathrm{CV}+\mathrm{N}$

COrE CV+N

Dust, $\nu_{\mathrm{cmb}}=70 \mathrm{GHz}$

Radiosources $30 \%$

Figure 12. BB APS for the reionization histories: suppression, filtering, late double peaked, early plus filtering for different values of tensor to scalar ratio $r$. Galactic synchrotron (dash-dotted blue line) and thermal dust (dash-triple-dotted cyan line) polarized emission, and extragalactic point source fluctuations (solid orange line) and their potential residual (solid violet line) as described in previous section (see plot legend and text for details).

Comparing the panels in figure it is clear that the ideal sensitivity of Planck is enough to detect the primordial B-mode for tensor-to-scalar ratios above few $\times 0.01$, in particularly thanks to the information contained in the reionization bump and up to the first acoustic peak. The improvement foreseen for an experiment with a sensitivity like COrE could allow to reveal the primordial B-mode polarization signal down to $r \simeq 0.001$ (or even lower).

The ultimate limitation comes from foregrounds. In the case of the B-mode, we show an estimate of the contamination by Galactic synchrotron and thermal dust polarized emission, and by extragalactic point source fluc- tuations, parametrized as described in the previous section. In all cases, the extragalactic signal is never dominant except at very high multipoles, but still remaining below the contribution by lensing, while Galactic foreground may significantly contaminate the CMB measure, especially at low and intermediate multipole.

The set of panels in Figure 13 presents the temperature-polarization cross-correlation APS, plotted in absolute value. The plus sign at the top of each panel denote positive correlation. Again, there is only a weak dependence on the $T / S$ parameter, and a substantial difference between the considered evolutionary models, in particular for the early plus filtering history up to $\ell \sim 400$. 

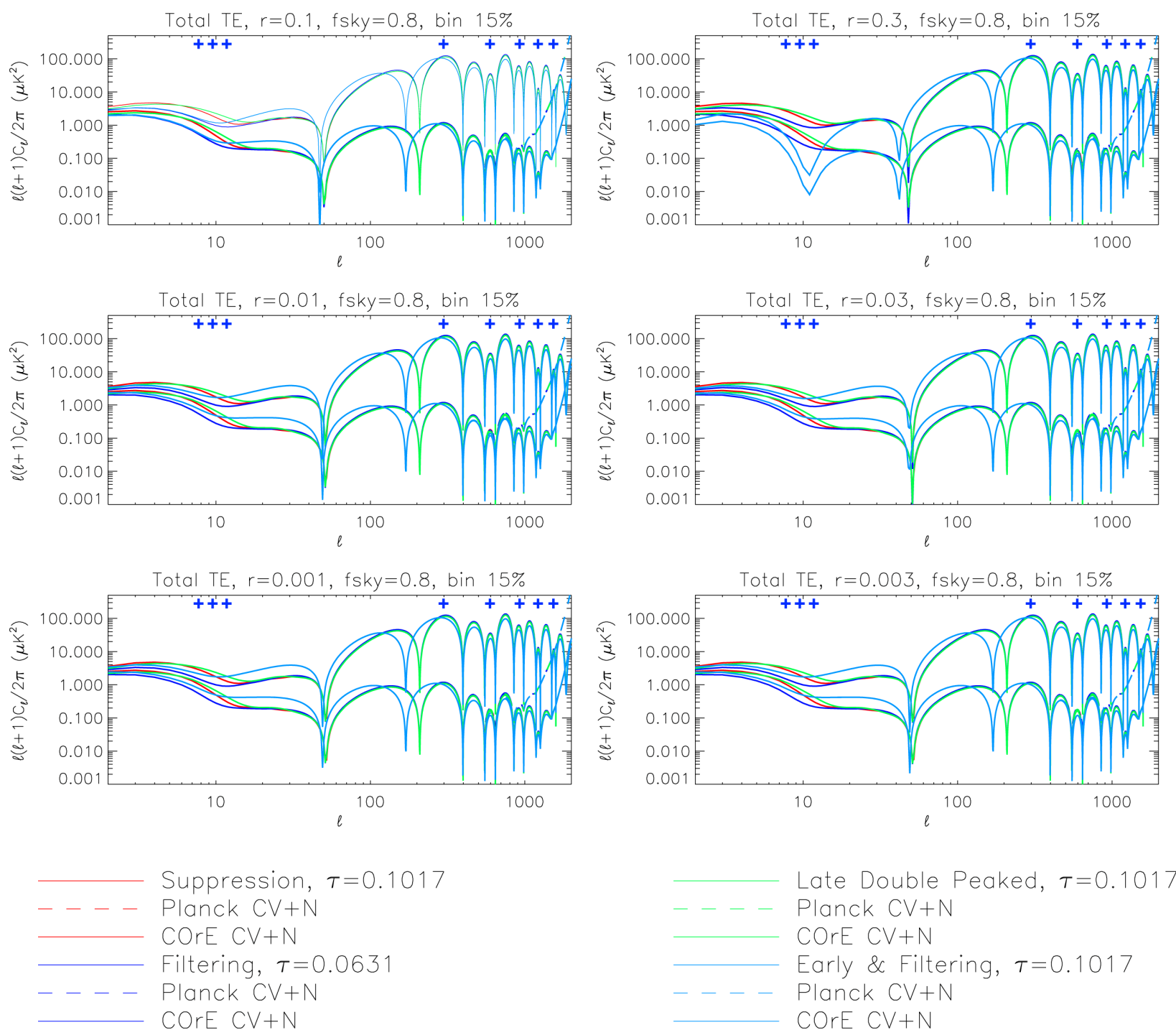

Figure 13. TE APS for the reionization histories: suppression, filtering, late double peaked, early plus filtering (see plot legend and text for details). Here is plotted the absolute value of the TE cross correlation, the "blue plus" indicates where the $C_{\ell}$ are positive and each cusp is an inversion point in the sign of the $C_{\ell}$ themselves.

\section{Comparison between Models}

In order to understand if different models can be distinguished, we analyze the relative differences between two models and compare them with experimental sensitivity and foreground residual estimates (properly normalized).

Since we are considering here several models, it is useful to define a reference model to which divide their differences as well as the experimental sensitivity and foreground limitation. We assume here a $\Lambda \mathrm{CDM}$ model with the same cosmological parameters adopted in the models under investigation, and an optical depth fixed by the suppression history $\left(\tau_{\text {Rif }}=\tau_{C F}\right)$. We compute its power spectra using the standard CAMB.

Note that, in principle, each model could be normalized in order to match available CMB data in the desired range of multipoles. Currently, temperature data play the major role in this respect. The overall normalization of the APS of a given model is related to the amplitude of initial perturbations, or, equivalently, to the density contrast at a reference scale, typically assumed at $8 \mathrm{~h}^{-1} \mathrm{Mpc}$, i.e. the parameter $\sigma_{8}$, which is better determined when CMB data are combined with galaxy surveys [66]. In practice, this involves a multiplicative factor of the APS. According to this choice, the relative differences between two models could be more or less prominent at different multipole ranges. In order to make our comparison between models dependent only on the APS shape and not on the normalization adopted for each model, before of computing their relative differences, we renormalize each model to make its TT APS averaged over $\ell$ equal to 
that of the reference model.

We report in Figures 14-17 the relative differences between models for the temperature anisotropies, polarization EE and BB APS, and the temperature-polarization cross-correlation, respectively. Each panel shows the comparison between two models, normalized to the reference model defined above, for a wide set of the $T / S$ parameter.

As anticipated, the tensor-to-scalar ratio does not affect significantly the temperature anisotropies, and for this reason in Figure 14 the curves appear almost superimposed.
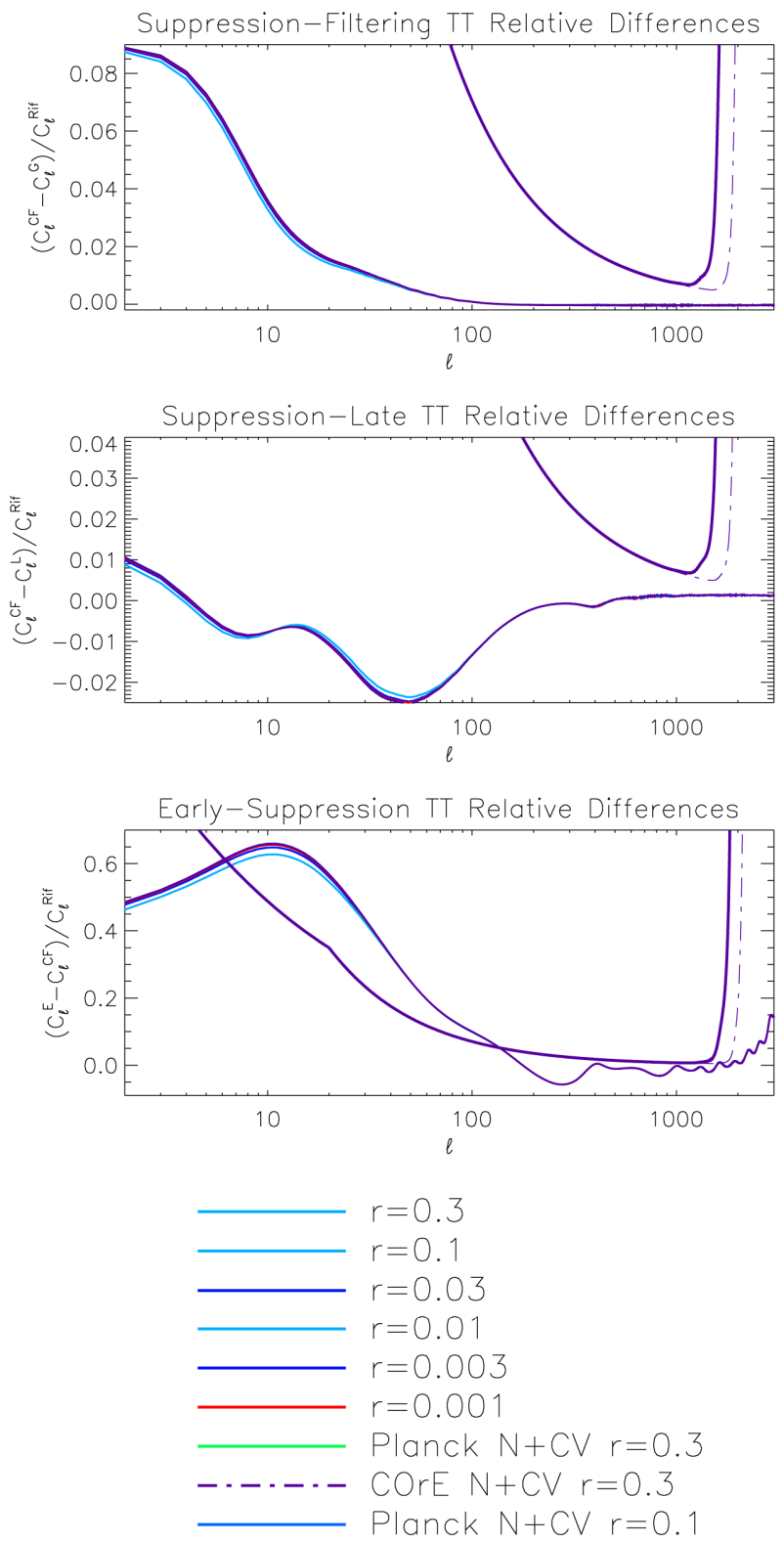

In addition, at $\ell \gtrsim 100-300$ the differences tend to be approximately null, except for the comparison of the early plus filtering model with all the others, because of their dramatic difference at high redshifts in the ionization fraction evolution.

Considering the whole multipole range, the largest differences appear again in the comparison of the early plus filtering model with all the other (later) histories, achieving a maximum level of $\sim 70 \%$ at $\ell \sim 10-15$.

Note that we can discriminate only early processes from other (later) models using only the CMB TT APS. This can not be significantly improved with the future
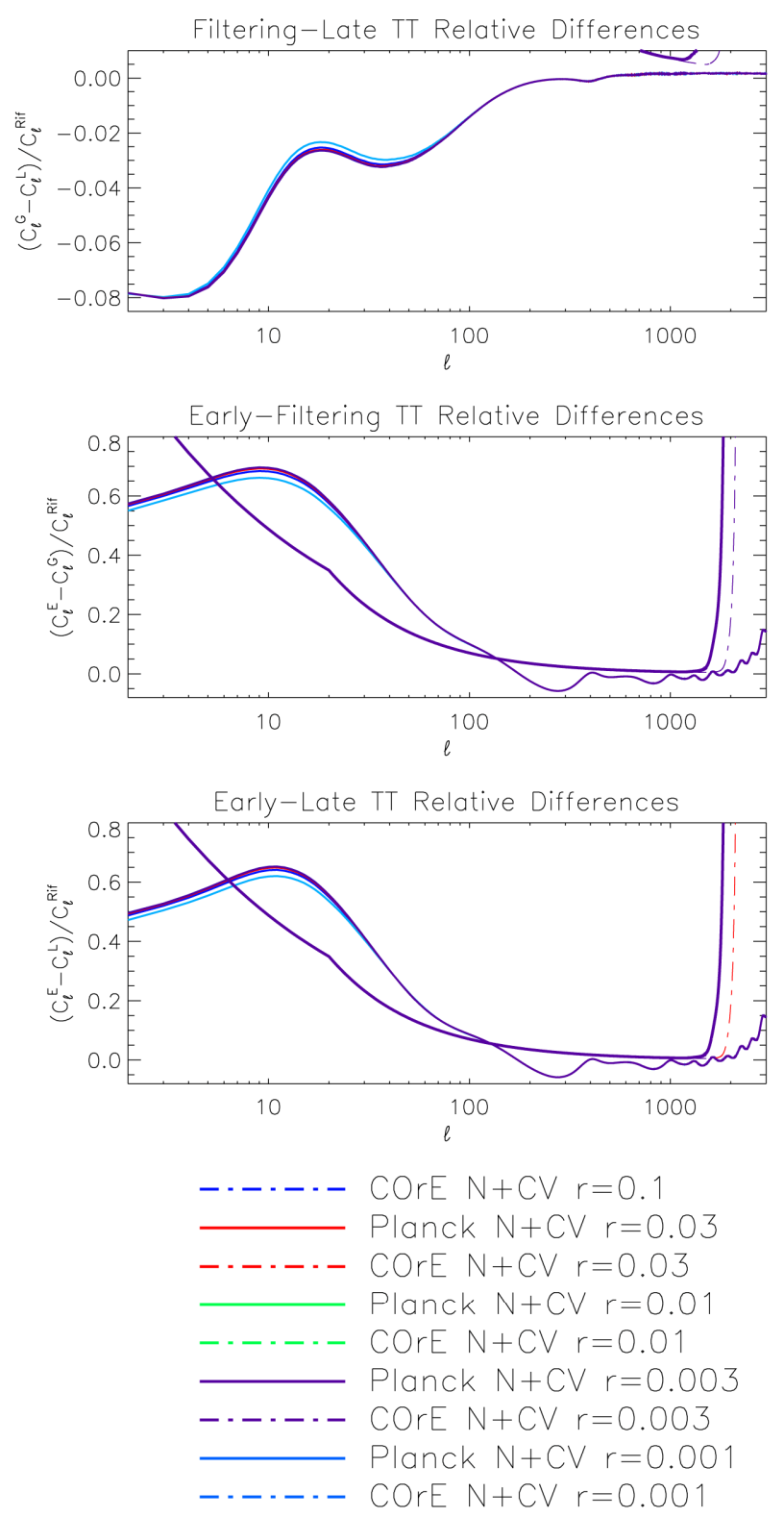

Figure 14. Relative differences between the astrophysical and phenomenological reionization histories in temperature power spectrum for all values of the $\boldsymbol{r}$ parameter assumed in this work (see plot legend and text for details). $C_{\ell}^{\text {Rif }}$ is the adopted normalization power spectrum. 

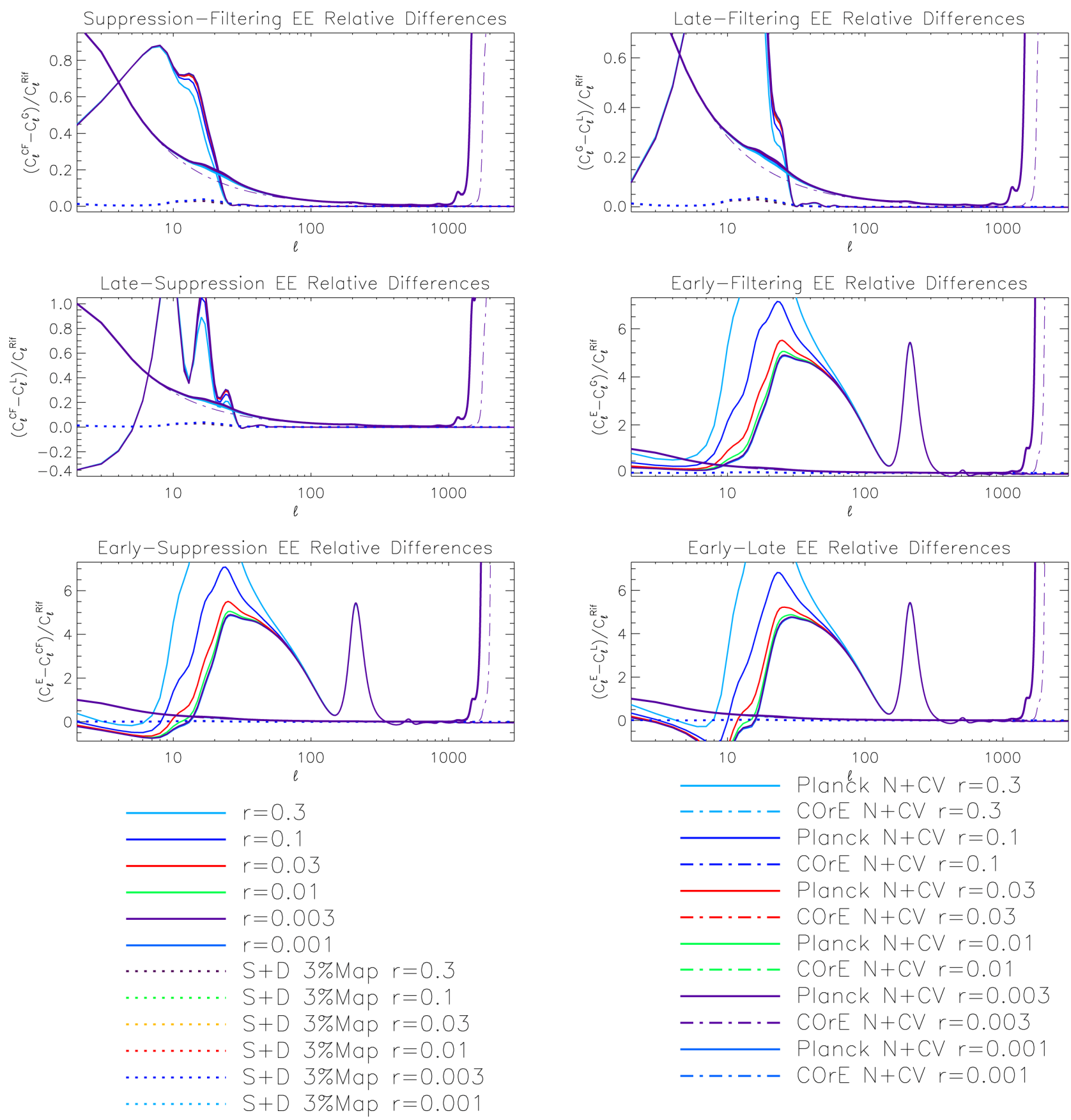

Figure 15. Relative differences between the astrophysical and phenomenological reionization histories in polarization EE-mode power spectrum for all values of the $r$ parameter assumed in this work. $C_{\ell}^{\text {Rif }}$ is the adopted normalization power spectrum. Potential residuals of Galactic foregrounds are also shown (see plot legend and text for details).

generation of experiments, the limitation coming essentially from cosmic variance. Thus, polarization data are crucial.

The comparison between the E-mode polarization power spectra is more interesting (see Figure 15). As evident, in particular in the three last panels in Figure 15 where the early plus filtering scenario is compared with the others, the differences related to the $r$ parameter emerge clearly. The greater is $r$ the greater are the relative differences. The large difference at $\ell \sim 200$ is due to the first early reionization phase, absent in the other models.

In general, remarkable relative differences appear between all the models at $\ell \sim 5-25$ or even up to $\ell \sim 100$ when comparing the early plus filtering scenario with the others. They are connected to the details of 

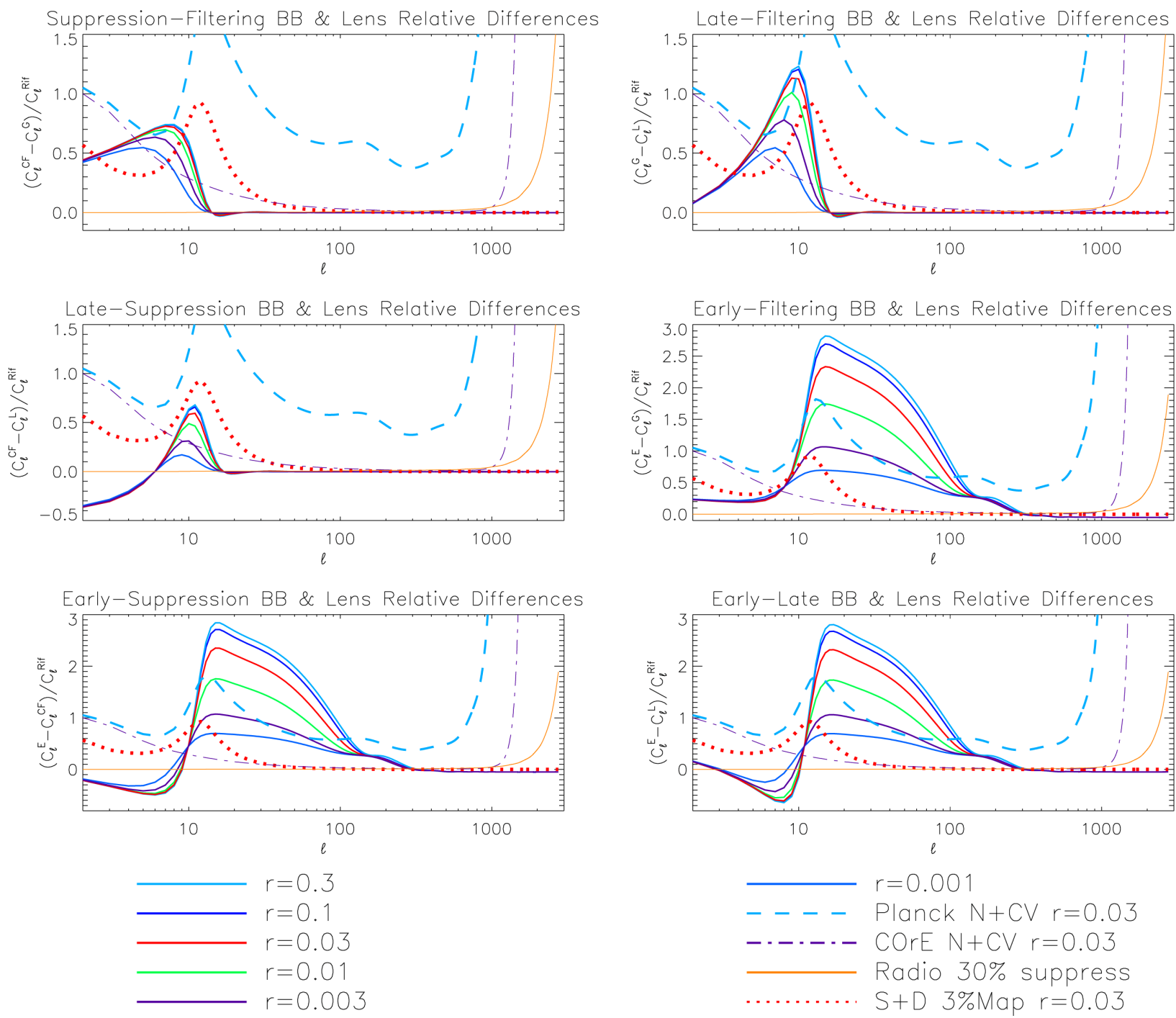

Figure 16. Relative differences between the astrophysical and phenomenological reionization histories in polarization B-mode power spectrum for all values of the $\boldsymbol{r}$ parameter assumed in this work. $C_{\ell}^{\text {Rif }}$ is the adopted normalization power spectrum. Potential residuals of Galactic foregrounds and extragalactic point source fluctuations are also shown (see plot legend and text for details).

the ionization history at lower redshifts. We also plot a potential residual contamination from Galactic foregrounds, $S+D$ in the plots. Note that a foreground removal at a few per cent level of accuracy at map level makes astrophysical contamination below the sensitivity limitation of on-going and future space experiments. The difference in nominal capability of Planck and COrE is not so remarkable in this respect, although, in practice, a significant improvement in sensitivity and frequency coverage clearly will make much more robust and accurate the foreground subtraction process.

More complex is the case of the B-mode polarization power spectra, reported in Figure 16, where, for completeness, we display also an estimate of the potential residual from extragalactic point source fluctuations which is always negligible in comparison with the other sources of limitation. For simplicity in these plots we also choose a reference tensor to scalar ratio, $r=0.03$, in order to represent the limit in the sensitivity of Planck and COrE in respect to the relative signals of the histories, and to avoid an excessive overlapping of the curves. The higher is its value, the bigger is the capability of the instruments to discriminate between the models giving a lower limiting curve. The same reference $T / S$ parameter was applied for the contamination due to synchrotron and dust signals. As before, the early plus filtering model largely differ from the others. Note that for the B-mode the relative differences between the various models remarkably depend on $r$ in all cases. The Planck sensitivity strikingly depends on $r$ at low and 

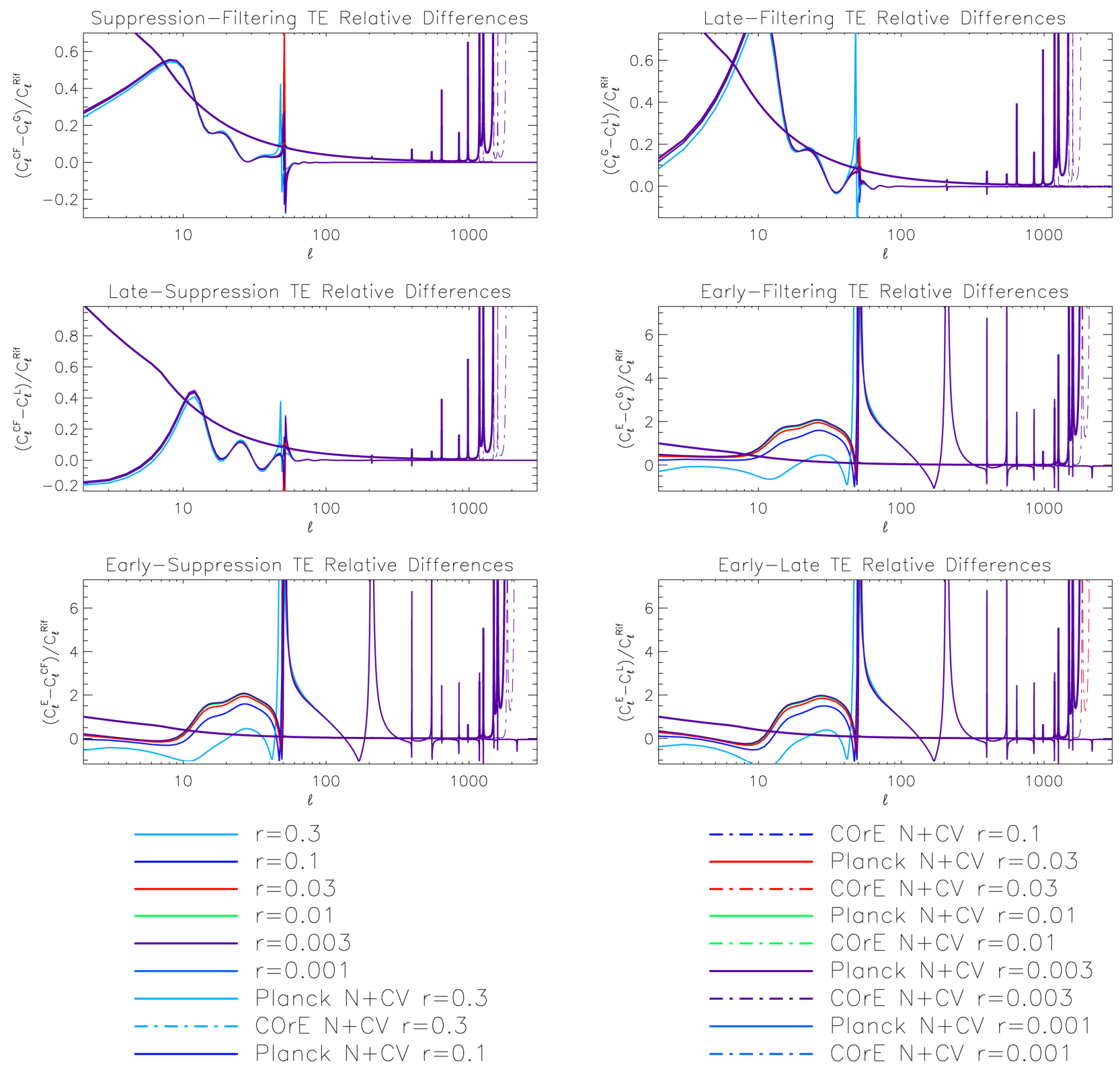

Figure 17. Relative differences between the astrophysical and phenomenological reionization histories in temperature polarization cross correlation power spectrum for all values of the $r$ parameter assumed in this work (see plot legend and text for details). $C_{\ell}^{\text {Rif }}$ is the adopted normalization power spectrum.

intermediate $\ell$, because of the instrumental noise limitation, while that of COrE is almost independent on $r$, being essentially cosmic variance limited where the lensing contribution does not dominate. The sensitivity of COrE is such that we could distinguish between suppression and filtering (or late) models in a certain range of multipoles (around $\ell \sim 10$ ) even for $r$ slightly larger than $\sim 10^{-3}$, while the early plus filtering history can be distinguished from all the other histories on a wider multipole region (or for even lower values of $r$ ). The impact of residuals of Galactic polarized foregrounds clearly increases for decreasing tensor-to-scalar ratio, as expected in the comparison of relative differences between models. Only for $r \gtrsim 0.1$ a foreground subtraction at $3 \%$ accuracy level in the map is enough to discriminate between each couple of considered models, while the early plus filtering history can be distinguished from all the other histories even with a less accurate foreground subtraction, thanks to its prominent differences at intermediate multipoles.

Finally, relative differences in the temperature-polarization cross-correlation APS are also very large on very wide ranges of multipole when comparing the early plus filtering history with all the other models (see Fig- 
ure 17), filtering and late models show remarkable differences (larger than $80 \%$ ) at low multipoles, while the suppression model differ from the late and filtering models only on a very small range of multipoles around $\ell \sim 10$. The "spikes" appearing at high multipoles are due to little shifts of the multipoles corresponding to the change of sign of the cross-correlation spectra for the different considered models. The difference in nominal capability of Planck and COrE is not so remarkable for the TE mode.

\section{Conclusions}

The inclusion of astrophysically motivated ionization and thermal histories in numerical codes is crucial for the accurate prediction of the features induced in the CMB, for constraining reionization models with $\mathrm{CMB}$ data, and to exploit current and future high quality $\mathrm{CMB}$ data with great versatility to accurately extract cosmological information.

We have implemented a modified version of CAMB, the Cosmological Boltzmann code for computing the APS of the anisotropies of the CMB, to introduce the hydrogen and helium ionization fractions predicted in two astrophysical reionization models, i.e. suppression and filtering model, in two classes of phenomenological reionization histories, involving late or early reionization, and in their combination, as alternative to the original implementation of reionization in the CAMB code, and beyond the simple $\tau$-parametrization. For astrophysical models, we provide also suitable analytical descriptions of the ionization and thermal histories that can be ingested in any numerical code aimed at computing CMB features. We compared the results obtained for these models for all the non-vanishing (in the assumed scenarios) modes of the CMB APS. As a significant step forward with respect to previous analyses, the emphasis has been posed here to the extension to a detailed characterization of the polarization B-mode APS for these classes of models. Its amplitude and shape depends, in particular, on the tensor-to-scalar ratio, $r$, and on the reionization history, thus an accurate modeling of the reionization process will have implications for the precise determination of $r$ or to set more precise constraints on it through the joint analysis of $\mathrm{E}$ and $\mathrm{B}$-mode polarization data available in the next future and from a mission of next generation. This is particularly crucial in the case of low values of $r$, because of the contribution to B-mode coming from lensing that competes with the primordial B-mode at intermediate multipoles. Taking into account also the limitation from potential residuals of astrophysical foregrounds, we discussed the capability of next data to disentangle between different reionization scenarios in a wide range of tensor-to-scalar ratios.

\section{Acknowledgements}

We acknowledge the use of the Legacy Archive for Microwave Background Data Analysis (LAMBDA). Support for LAMBDA is provided by the NASA Office of Space Science. We warmly thank Enrico Franceschi for his technical advices in TeX compilation. We acknowledge support by ASI through ASI/INAF Agreement I/072/09/0 for the Planck LFI Activity of Phase E2 and by MIUR through PRIN 2009 grant no. 2009XZ54H2.

\section{REFERENCES}

[1] P. J. E. Peebles, "Recombination of the Primeval Plasma," The Astrophysical Journal, Vol. 153, 1968, p. 1. doi: 10.1086/149628

[2] Ya. B. Zel'dovich, V. Kurt and R. A. Sunyaev, "Recombination of Hydrogen in the Hot Model of the Universe," Zhurnal Eksperimental'noi i Teoreticheskoi Fiziki, Vol. 55, No. 1, 1968, pp. 278-286.

[3] B. J. T. Jones and R. Wyse, "The Ionisation of the Primeval Plasma at the Time of Recombination," Astronomy \& Astrophysics, Vol. 149, No. 1, 1985, p. 144.

[4] S. Seager, D. D. Sasselov and D. Scott, "How Exactly Did the Universe Become Neutral?" The Astrophysical Journal Supplement Series, Vol. 128, No. 2, 2000, pp. 407430, arXiv:astro-ph/9912182. doi:10.1086/313388

[5] N. A. Zabotin and P. D. Naselsky, "The Neutrino Background in the Early Universe and Temperature Fluctuations in the Cosmic Microwave Radiation," Soviet Astronomy, Vol. 26, 1982, p. 272.

[6] E. R. Switzer and C. M. Hirata, "Primordial Helium Recombination. III. Thomson Scattering, Isotope Shifts, and Cumulative Results," Physical Review D, Vol. 77, No. 8, 2008, 3008, arXiv:astro-ph/0702145. doi:10.1103/PhysRevD.77.083008

[7] A. A. Starobinsky, "Spectrum of Relict Gravitational Radiation and the Early State of the Universe," Journal of Experimental and Theoretical Physics Letters, Vol. 30, No. 11, 1979, pp. 682-685.

[8] L. F. Abbott and M. B. Wise, "Constraints on Generalized Inflationary Cosmologies," Nuclear Physics B, Vol. 244, No. 2, 1984, pp. 541-548. doi:10.1016/0550-3213(84)90329-8

[9] A. R. Liddle and D. H. Lyth, "COBE, Gravitational Waves, Inflation and Extended Inflation," Physics Letters B, Vol. 291, 1992, pp. 391-398, arXiv:astro-ph/9208007. doi:10.1016/0370-2693(92)91393-N

[10] A. R. Liddle and D. H. Lyth, "The Cold Dark Matter Density Perturbations," Physics Reports, Vol. 231, No. 1-2, 1993, pp. 1-105, arXiv:astro-ph/9303019. doi:10.1016/0370-1573(93)90114-S

[11] S. Dodelson, "Modern Cosmology," Academic Press, San Francisco, 2003.

[12] D. N. Spergel, et al., "Three-Year Wilkinson Microwave Anisotropy Probe (WMAP) Observations: Implications for Cosmology," The Astrophysical Journal Supplement 
Series, Vol. 170 , No. 2, 2007, p. 377 , arXiv:astro-ph/06 03449 .

[13] J. Dunkley, et al., "Five-Year Wilkinson Microwave Anisotropy Probe Observations: Likelihoods and Parameters from the WMAP Data," The Astrophysical Journal Supplement Series, Vol. 180, No. 2, 2009, pp. 306-329, arXiv: astro-ph/0803.0586.

[14] E. Komatsu, et al., "Five-Year Wilkinson Microwave Anisotropy Probe Observations: Cosmological Interpretation," The Astrophysical Journal Supplement Series, Vol. 180, No. 2, 2009, p. 330, arXiv:astro-ph/0803.0547.

[15] D. Larson, et al., "Seven-Year Wilkinson Microwave Anisotropy Probe (WMAP) Observations: Power Spectra and WMAP-derived Parameters," The Astrophysical Journal Supplement Series, Vol. 192, No. 2, 2011, arXiv:astro-ph/1001.4635.

[16] E. Komatsu, et al., "Seven-year Wilkinson Microwave Anisotropy Probe (WMAP) Observations: Cosmological Interpretation," The Astrophysical Journal Supplement Series, Vol. 192, No. 2, 2011, arXiv:astro-ph/1001.4538.

[17] R. Cen, "The Universe Was Reionized Twice," The Astrophysical Journal, Vol. 591, 2003, pp. 12-37, arXiv: astro-ph/0210473.

[18] B. Ciardi, A. Ferrara and S. M. D. White, "Early Reionization by the First Galaxies," Monthly Notices of the Royal Astronomical Society, Vol. 344, No. 1, 2003, pp. L7-L11, arXiv:astro-ph/0302451. doi:10.1046/j.1365-8711.2003.06976.x

[19] A. G. Doroshkevich and P. D. Naselsky, "Ionization History of the Universe as a Test for Superheavy Dark Matter particles," Physical Review D, Vol. 65, No. 12, 2002, Article ID: 13517, arXiv:astro-ph/0201212.

[20] A. G. Doroshkevich, I. P. Naselsky, P. D. Naselsky and I. D. Novikov, "Ionization History of the Cosmic Plasma in the Light of the Recent Cosmic Background Imager and Future Planck Data," The Astrophysical Journal, Vol. 586, No. 2, 2002, p. 709, arXiv:astro-ph/0208114. doi:10.1086/367819

[21] S. H. Hansen and Z. Haiman, "Do We Need Stars to Reionize the Universe at High Redshifts? Early Reionization by Decaying Heavy Sterile Neutrinos," The Astrophysical Journal, Vol. 600, No. 1, 2004, pp. 26-31, arXiv: astro-ph/0305126. doi:10.1086/379636

[22] S. Kasuya, M. Kawasaki and N. Sugiyama, "Partially Ionizing the Universe by Decaying Particles," Physical Review D, Vol. 69, No. 2, 2004, Article ID: 023512, arXiv: astro-ph/0309434.

[23] P. J. E. Peebles, S. Seager and W. Hu, "Delayed Recombination," The Astrophysical Journal, Vol. 539, 2000, pp. L1-L4, arXiv:astro-ph/0004389. doi:10.1086/312831

[24] L. A. Popa, C. Burigana and N. Mandolesi, "Radiative Effects by High-z UV Radiation Background: Implications for the Future CMB Polarization Measurements," New Astronomy, Vol. 11, No. 3, 2005, pp. 173-184, arXiv: astro-ph/0506454. doi:10.1016/j.newast.2005.07.003

[25] J. S. B. Wyithe and R. Cen, "The Extended Star Formation History of the First Generation of Stars and the Reionization of Cosmic Hydrogen," The Astrophysical
Journal, Vol. 659, No. 2, 2007, pp. 890-907. doi: $10.1086 / 511948$

[26] C. Burigana, F. Finelli, R. Salvaterra, L. A. Popa and N. Mandolesi, "On the Cosmological Implications of Next and future CMB Space Experiments," Recent Research Developments in Astronomy \& Astrophysics, Vol. 2, 2004, pp. 59-117.

[27] R. Schneider, R. Salvaterra, T. R. Choudhury, A. Ferrara, C. Burigana and L. A. Popa, "Detectable Signatures of Cosmic Radiative Feedback," Monthly Notice of the Royal Astronomical Society, Vol. 384, No. 4, 2008, pp. 15251532, arXiv:0712.0538.

[28] C. Burigana, L. A. Popa, R. Salvaterra, R. Schneider, C. T. Roy and A. Ferrara, "Cosmic Microwave Background Polarization Constraints on Radiative Feedback," Monthly Notice of the Royal Astronomical Society, Vol. 385, No. 1, 2008, pp. 404-410, arXiv:0712.1913.

[29] P. Naselsky and L. Y. Chiang, "Late Reionizations of the Universe and Their Manifestation in the WMAP and Future Planck Data," Monthly Notice of the Royal Astronomical Society, Vol. 347, No. 3, 2004, pp. 921-936, arXiv: astro-ph/0302085.

[30] U. Seljak and M. Zaldarriaga, "Signature of Gravity Waves in the Polarization of the Microwave Background," Physical Review Letters, Vol. 78, No. 11, 1997, pp. 2054-2057, arXiv:astro-ph/9609169. doi:10.1103/PhysRevLett.78.2054

[31] M. Kamionkowski and A. Kosowsky, "Detectability of Inflationary Gravitational Waves with Microwave Background Polarization," Physical Review D, Vol. 57, No. 2, 1998, pp. 685-691, arXiv:astro-ph/9705219. doi:10.1103/PhysRevD.57.685

[32] W. Hu, "Reionization Revisited: Secondary Cosmic Microwave Background Anisotropies and Polarization," The Astrophysical Journal, Vol. 529, 2000, p. 12, arXiv:astro-ph/9907103.

[33] U. Seljak and C. M. Hirata, "Gravitational Lensing as a Contaminant of the Gravity Wave Signal in the CMB," Physical Review D, Vol. 69, No. 4, 2004, Article ID: 043005, arXiv:astro-ph/0310163. doi:10.1103/PhysRevD.69.043005

[34] R. Salvaterra, A. Ferrara and P. Dayal, "Simulating HighRedshift Galaxies," Monthly Notice of the Royal Astronomical Society, Vol. 414, No. 2, 2011, pp. 847-859, arXiv: astro-ph/1003.3873.

[35] The Planck Collaboration, "The Scientific Programme of Planck," ESA-SCI(2005)1, 2005, arXiv:astro-ph/0604069.

[36] The COrE Collaboration, "COrE (Cosmic Origins Explorer) A White Paper," 2011, arXiv:astro-ph/1102.2181.

[37] R. Salvaterra, B. Ciardi, A. Ferrara and C. Baccigalupi, "Reionization History from Coupled Cosmic Microwave Background/21-cm Line Data," Monthly Notice of the Royal Astronomical Society, Vol. 360, No. 3, 2005, pp. 1063-1068, arXiv:astro-ph/0502419.

[38] I. T. Iliev, U.-L. Pen, J. R. Bond, G. Mellema and P. R. Shapiro, "The Kinetic Sunyaev-Zel'dovich Effect from Radiative Transfer Simulations of Patchy Reionization," The Astrophysical Journal, Vol. 660, No. 2, 2007, pp. 
933-944, arXiv:astro-ph/0609592. doi:10.1086/513687

[39] R. A. Sunyaev and Ya. B. Zel'dovich, "The Interaction of Matter and Radiation in the Hot Model of the Universe II," Astrophysics and Space Science, Vol. 4, No. 3, 1970, pp. 301-316.

[40] Ya. B. Zel'dovich, A. F. Illarionov and R. A. Sunyaev, "Influence of Energy Release on the Radiation Spectrum in the Hot Model of the Universe," Zhurnal Eksperimental'noi i Teoreticheskoi Fiziki, Vol. 68, 1972, p.1217.

[41] Ya. B. Zel'dovich and R. A. Sunyaev, "The Interaction of Matter and Radiation in a Hot-Model Universe," Astrophysics and Space Science, Vol. 4, No. 3, 1969, pp. 301316. doi:10.1007/BF00661821

[42] P. Procopio and C. Burigana, "A Numerical Code for the Solution of the Kompaneets Equation in Cosmological Context," Astronomy \& Astrophysics, Vol. 507, No. 3, 2009, pp. 1243-1256, arXiv:0905.2886. doi:10.1051/0004-6361/200912061

[43] D. J. Fixsen, E. S. Cheng, J. M. Gales, J. C. Mather, R. A. Shafer and E. L. Wright, "The Cosmic Microwave Background Spectrum from the Full COBE FIRAS Data Set," The Astrophysical Journal, Vol. 473, No. 2, 1996, pp. 576587, arXiv:astro-ph/9605054. doi:10.1086/178173

[44] J. C. Mather, D. J. Fixsen, R. A. Shafer, C. Mosier and D. T. Wilkinson, "Calibrator Design for the COBE Far-Infrared Absolute Spectrophotometer (FIRAS)," The Astrophysical Journal, Vol. 512, No. 2, 1999, p. 511, arXiv: astro-ph/9810373. doi:10.1086/306805

[45] X. Fan, et al., "Constraining the Evolution of the Ionizing Background and the Epoch of Reionization with z 6 Quasars. II. A Sample of 19 Quasars," The Astronomical Journal, Vol. 132, No. 1, 2006, pp. 117-136, arXiv:astro-ph/051 2082.

[46] S. Gallerani, T. R. Choudhury and A. Ferrara, "Constraining the Reionization History with QSO Absorption Spectra," Monthly Notice of the Royal Astronomical Society, Vol. 370, No. 3, 2006, pp. 1401-1421, arXiv:astro-ph/0512129.

[47] N. Gnedin and X. Fan, "Cosmic Reionization Redux," The Astrophysical Journal, Vol. 648, No. 1, 2006, p. 1, arXiv:astro-ph/0603794. doi:10.1086/505790

[48] T. R. Choudhury and A. Ferrara, "Experimental Constraints on Self-Consistent Reionization Models," Monthly Notice of the Royal Astronomical Society, Vol. 361, No. 2, 2005, pp. 577-594, arXiv:astro-ph/0411027.

[49] T. R. Choudhury and A. Ferrara, "Updating Reionization Scenarios after Recent Data," Monthly Notice of the Royal Astronomical Society, Vol. 371, No. 1, 2006, pp. L55-L59, arXiv:astro-ph/0603617.

[50] J. Miralda-Escudé, M. Haehnelt and M. J. Rees, "Reionization of the Inhomogeneous Universe," The Astrophysical Journal, Vol. 530, No. 1, 2000, p. 1, arXiv:astro-ph/98 12306.

[51] R. Schneider, R. Salvaterra, A. Ferrara and B. Ciardi, "Constraints on the Initial Mass Function of the First Stars," Monthly Notice of the Royal Astronomical Society, Vol. 369, No. 2, 2006, pp. 825-834, arXiv:astro-ph/051 0685 .

[52] R. H. Kramer, Z. Haiman and S. P. Oh, "Feedback from
Clustered Sources during Reionization," The Astrophysical Journal, Vol. 649, 2006, pp. 570-578, arXiv:astro-ph/ 0604218. doi:10.1086/506906

[53] Z. Haiman and G. L. Bryan, "Was Star Formation Suppressed in High-Redshift Minihalos?" The Astrophysical Journal, Vol. 650, 2006, pp. 7-11, arXiv:astro-ph/0603 541.

[54] M. A. Alvarez, P. R. Shapiro, K. Ahn and I. T. Iliev, "Implications of WMAP 3 Year Data for the Sources of Reionization," The Astrophysical Journal, Vol. 644, No. 2, 2006, p. L101, arXiv:astro-ph/0604447. doi:10.1086/505644

[55] N. Gnedin, "Effect of Reionization on Structure Formation in the Universe," The Astrophysical Journal, Vol. 542, No. 2, 2000, pp. 535-541, arXiv:astro-ph/0002151. doi: $10.1086 / 317042$

[56] WaveMetrics Inc. 2010, Igor Pro (version 6.2).

[57] A. Lewis, A. Challinor and A. Lasenby, "Efficient Computation of Cosmic Microwave Background Anisotropies in Closed Friedmann-Robertson-Walker Models," The Astrophysical Journal, Vol. 538, No. 2, 2000, pp. 473476, arXiv:astro-ph/9911177. doi:10.1086/309179

[58] U. Seljak and M. Zaldarriaga, "A Line-of-Sight Integration Approach to Cosmic Microwave Background Anisotropies," The Astrophysical Journal, Vol. 469, 1996, pp. 437-444, arXiv:astro-ph/9603033. doi:10.1086/177793

[59] T. Trombetti and C. Burigana, "Fitting Ionization Fraction and Electron Temperature Histories for Astrophysical Reionization Models," Internal Report IASF-BO 589/ 2011.

[60] L. Knox, "Determination of Inflationary Observables by Cosmic Microwave Background Anisotropy Experiments," Physical Review D, Vol. 52, No. 8, 1995, pp. 4307-4318, arXiv:astro-ph/9504054. doi:10.1103/PhysRevD.52.4307

[61] L. Page, et al., "Three-Year Wilkinson Microwave Anisotropy Probe (WMAP) Observations: Polarization Analysis," The Astrophysical Journal Supplement Series, Vol. 170, No. 2, 2007, pp. 335-376, arXiv:astro-ph/0603450. doi:10.1086/513699

[62] L. Toffolatti, G. F. Argüeso, G. de Zotti, P. Mazzei, A. Franceschini, L. Danese and C. Burigana, "Extragalactic Source Counts and Contributions to the Anisotropies of The Cosmic Microwave Background: Predictions for the Planck Surveyor Mission," Monthly Notice of the Royal Astronomical Society, Vol. 297, No. 1, 1998, pp. 117-127, arXiv:astro-ph/9711085.

[63] G. de Zotti, R. Ricci, D. Mesa, L. Silva, P. Mazzotta, L. Toffolatti and J. González-Nuevo, "Predictions for HighFrequency Radio Surveys of Extragalactic Sources," Astronomy \& Astrophysics, Vol. 431, No. 3, 2005, pp. 893903, arXiv:astro-ph/0410709.

[64] M. Tucci and L. Toffolatti, "The Impact of Polarized Extragalactic Radio Sources on the Detection of CMB Anisotropies in Polarization," Advances in Astronomy, 2012, Article ID: 624987, arXiv:astro-ph/1204.0427.

[65] P. de Bernardis, M. Bucher, C. Burigana and L. Piccirillo, "B-Pol: Detecting Primordial Gravitational Waves Generated during Inflation," Experimental Astronomy, Vol. 23, No. 1, 2009, pp. 5-16, arXiv:astro-ph/0808.1881. 
[66] L. A. Popa, C. Burigana and N. Mandolesi, "Cosmological Parameter Determination from Planck and Sloan Digital Sky Survey Data in $\Lambda$ Cold + Hot Dark Matter Cos- mologies," The Astrophysical Journal, Vol. 558, No. 1, 2001, pp. 10-22, arXiv:astro-ph/0102138.

$\underline{\text { doi: } 10.1086 / 322448}$ 


\section{Appendix}

\section{A. Fitting Ionization and Thermal Histories}

The considered astrophysical reionization models, as well as others published in the literature, provide ionization and thermal histories in tabulated form. The cosmological analysis of CMB anisotropy data largely relies on Boltzmann codes for computing the angular power spectrum in temperature, polarization, and cross-correlation modes under general conditions. The inclusion in such codes of reionization histories beyond the simplistic phenomenological approximations already implemented in the publicly available codes, allows to achieve more accurate predictions, of particular interest for the analysis of polarization data. Having functional descriptions of the evolution of the ionization fraction allows to speedup computation and improves code versatility with respect to the use of interpolation of tabulated grids. Although in this context only the ionization history is relevant, we report here for completeness also the results concerning the thermal history.

The software we used to fit to the suppression and filtering model is Igor Pro (v. 6.21), an integrated program for visualizing, analyzing, transforming and presenting experimental data, such as curve-fitting, Fourier transforms, smoothing, statistics, and other data analysis, image display and processing, by a combination of graphical and command-line user interface. For completeness, we report here all the functional forms we found particularly suitable to represent the considered ionization and thermal histories. Their compilation could be also useful as guideline to fit other kinds of reionization history. More details about the usage of this software to the current aims and the complete list of parameters of the below functions are given in [59].

\section{A.1. Fitting Functions for the Reionization Histories}

We report here below the redshift intervals and the corresponding analytic functions found in the case of the suppression model.

- $z=[0,3.8]$-Polinomial Function of $6^{\circ}$ order:

$$
\begin{aligned}
& \chi_{r e}=\sum_{i=0}^{6} \chi_{i} z^{i} \quad \text { with coefficients } \\
& \chi_{0}=1.12751, \chi_{1}=0.082246, \\
& \chi_{2}=-0.083182, \chi_{3}=0.041472, \\
& \chi_{4}=-0.010615, \chi_{5}=0.0012916, \\
& \chi_{6}=-5.5608 \times 10^{-5} .
\end{aligned}
$$

- $z=[3.8,6]$-Polinomial Function of $5^{\circ}$ order:

$$
\begin{aligned}
& \chi_{\text {re }}=\sum_{i=0}^{5} \chi_{i} z^{i} \quad \text { with coefficients } \\
& \chi_{0}=-14.708, \quad \chi_{1}=16.69,
\end{aligned}
$$

$$
\begin{aligned}
& \chi_{2}=-6.8995, \chi_{3}=1.4055 \\
& \chi_{4}=-0.14167, \chi_{5}=0.0056647 .
\end{aligned}
$$

- $z=[6,9]$-Polinomial Function of $5^{\circ}$ order, with coefficients:

$$
\begin{aligned}
& \chi_{0}=-8.6358, \chi_{1}=6.7374, \chi_{2}=-1.821, \\
& \chi_{3}=0.24108, \chi_{4}=-0.015686, \chi_{5}=0.00040015 .
\end{aligned}
$$

- $\quad z=[9,12.4]-$ Log-Normal Function:

$$
\chi_{r e}=\chi_{0}+\chi_{1} \exp \left[-\left(\frac{\ln \left(z / \chi_{2}\right)}{\chi_{3}}\right)^{2}\right] \text { with coefficients }
$$

$\chi_{0}=1.0061, \chi_{1}=-0.83887$,

$\chi_{2}=14.107, \chi_{3}=0.26413$.

- $\quad z=[12.4,14.2]$-Sigmoidal Function:

$$
\begin{aligned}
& \chi_{r e}=\chi_{0}+\frac{\chi_{1}}{1+\exp \left(\frac{\chi_{2}-z}{\chi_{3}}\right)} \text { with coefficients } \\
& \chi_{0}=1.1468, \chi_{1}=-1.153, \\
& \chi_{2}=11.39, \chi_{3}=1.2238 .
\end{aligned}
$$

- $\quad z=[14.2,16.8]-$ Hill Equation:

$$
\begin{aligned}
& \chi_{r e}=\chi_{0}+\left(\chi_{1}-\chi_{0}\right) \frac{z^{\chi_{2}}}{z^{\chi_{2}}+\chi_{3}^{\chi_{2}}} \text { with coefficients } \\
& \chi_{0}=-0.0021632, \chi_{1}=0.46249, \\
& \chi_{2}=-13.862, \chi_{3}=12.952 .
\end{aligned}
$$

- $\quad Z=[16.8,18]$-Decaying Exponential Function (expXOffset):

$$
\begin{aligned}
& \chi_{\text {re }}=\chi_{0}+\chi_{1} \exp \left(\frac{\chi_{3}-z}{\chi_{2}}\right) \text { with coefficients } \\
& \chi_{0}=7.2284 \cdot 10^{-6}, \chi_{1}=0.010169, \\
& \chi_{2}=1.054, \chi_{3}=16.8 .
\end{aligned}
$$

- $\quad Z=[18,20]-$ Hill Equation, with coefficients:

$$
\begin{aligned}
& \chi_{0}=0.00048712, \chi_{1}=0.013733, \\
& \chi_{2}=-24.474, \chi_{3}=17.051 .
\end{aligned}
$$

- $\quad Z=[20,20.2]$ - Linear Function:

$$
\begin{aligned}
& \chi_{r e}=\chi_{0}+\chi_{1} z \text { with coefficients } \\
& \chi_{0}=0.0052133, \chi_{1}=-0.000223 .
\end{aligned}
$$

- $\quad z=[20.2,23]$-Decaying Exponential Function (expXOffset), with coefficients:

$$
\begin{aligned}
& \chi_{0}=0.00023877, \chi_{1}=0.00047086, \\
& \chi_{2}=0.85918, \chi_{3}=20.2 .
\end{aligned}
$$

- $z=[23,30]$ - Log-Normal Function, with coefficients: 


$$
\begin{aligned}
& \chi_{0}=0.00025689, \chi_{1}=-9.6891 \times 10^{-6}, \\
& \chi_{2}=25.421, \chi_{3}=0.10111 .
\end{aligned}
$$

We report here below the redshift intervals and the corresponding analytic functions found in the case of the filtering model.

- $z=[0,3.8]$-Polinomial Function of $9^{\circ}$ order:

$$
\begin{aligned}
& \chi_{r e}=\sum_{i=0}^{9} \chi_{i} z^{i} \quad \text { with coefficients } \\
& \chi_{0}=1.1245, \chi_{1}=0.10495, \\
& \chi_{2}=-0.19535, \chi_{3}=0.25489, \\
& \chi_{4}=-0.20598, \chi_{5}=0.098856, \\
& \chi_{6}=-0.028032, \chi_{7}=0.0045895, \\
& \chi_{8}=-0.00040036, \quad \chi_{9}=1.4397 \times 10^{-5} .
\end{aligned}
$$

- $\quad z=[3.8,6]-$ Log-Normal Function, with coefficients:

$$
\begin{aligned}
& \chi_{0}=1.8818, \chi_{1}=-0.7941, \\
& \chi_{2}=6.3915, \chi_{3}=1.7469 .
\end{aligned}
$$

- $\quad z=[6,6.2]$-Linear Function, with coefficients:

$$
\chi_{0}=2.2768, \chi_{1}=-0.1981 \text {. }
$$

- $\quad z=[6.2,9]-$ Hill Equation, with coefficients:

$$
\begin{aligned}
& \chi_{0}=-0.1805, \chi_{1}=2.7323, \\
& \chi_{2}=-3.6558, \chi_{3}=5.6876 .
\end{aligned}
$$

- $\quad z=[9,11.6]$-Sigmoidal Function, with coefficients:

$$
\begin{aligned}
& \chi_{0}=1.1438, \quad \chi_{1}=-1.1441, \\
& \chi_{2}=7.3904, \chi_{3}=1.4126 .
\end{aligned}
$$

- $\quad z=[11.6,13]$-Power Function:

$$
\begin{aligned}
& \chi_{\text {re }}=\chi_{0}+\chi_{1} z^{\chi_{2}} \quad \text { with coefficients } \\
& \chi_{0}=-0.0089383, \chi_{1}=2.0529 \times 10^{6}, \\
& \chi_{2}=-7.0525 .
\end{aligned}
$$

- $\quad z=[13,15]$-Power Function, with coefficients: $\chi_{0}=-0.0015104, \chi_{1}=9.1637 \times 10^{8}, \chi_{2}=-9.5468$.

- $\quad z=[15,17]$-Decaying Exponential Function (expXOffset), with coefficients:

$$
\begin{aligned}
& \chi_{0}=0.00013226, \chi_{1}=0.003812, \\
& \chi_{2}=1.1503, \chi_{3}=15 .
\end{aligned}
$$

- $\quad z=[17,19.6]$-Decaying Exponential Function (expXOffset), with coefficients:

$$
\begin{aligned}
& \chi_{0}=0.00023759, \chi_{1}=0.00057014, \\
& \chi_{2}=0.98278, \chi_{3}=17 .
\end{aligned}
$$

- $\quad z=[19.6,22.2]$-Decaying Exponential Function (expXOffset), with coefficients:

$$
\begin{aligned}
& \chi_{0}=0.00023514, \chi_{1}=4.6107 \cdot 10^{-5}, \\
& \chi_{2}=1.3061, \chi_{3}=19.6 .
\end{aligned}
$$

- $\quad z=[22.2,30]$-Decaying Exponential Function (expXOffset), with coefficients:

$$
\begin{aligned}
& \chi_{0}=0.052228, \chi_{1}=-0.051987, \\
& \chi_{2}=26607, \chi_{3}=22.2 .
\end{aligned}
$$

\section{A.2. Fitting Functions for the Temperature Histories} We report here below the redshift intervals and the corresponding analytic functions found in the case of the suppression model.

- $\quad z=[0,3.8]$-Polinomial Function of $8^{\circ}$ order:

$T_{r e}=\sum_{i=0}^{8} T_{i} z^{i}$ with coefficients

$T_{0}=3870, T_{1}=6793.7, T_{2}=7221.8$,

$T_{3}=-15962, T_{4}=13644, T_{5}=-6419.7$,

$T_{6}=1734.7, T_{7}=-252.65, T_{8}=15.379$.

- $\quad z=[3.8,5.8]$-Sigmoidal Function:

$$
\begin{aligned}
& T_{r e}=T_{0}+\frac{T_{1}}{1+\exp \left(\frac{T_{2}-z}{T_{3}}\right)} \text { with coefficients } \\
& T_{0}=20934, T_{1}=-8982.3, \\
& T_{2}=4.0373, T_{3}=0.45319 .
\end{aligned}
$$

- $\quad z=[5.8,13]$ - Hill Eqation:

$$
\begin{aligned}
& T_{r e}=T_{0}+\left(T_{1}-T_{0}\right) \frac{z^{T_{2}}}{z^{T_{2}}+T_{3}^{T_{2}}} \text { with coefficients } \\
& T_{0}=-204.2, T_{1}=12105, \\
& T_{2}=-10.529, T_{3}=11.612 .
\end{aligned}
$$

- $\quad Z=[13,16]-$ Hill Equation, with coefficients:

$$
\begin{aligned}
& T_{0}=-60.289, T_{1}=8498.4, \\
& T_{2}=-12.484, T_{3}=12.23 .
\end{aligned}
$$

- $\quad z=[16,16.8]$-Decaying Exponential Function (expXOffset):

$$
\begin{aligned}
& T_{r e}=T_{0}+T_{1} \exp \left(\frac{T_{3}-z}{T_{2}}\right) \text { with coefficients } \\
& T_{0}=0.78206, T_{1}=229.94, T_{2}=1.0994, T_{3}=16 .
\end{aligned}
$$

- $\quad Z=[16.8,18.6]-$ Hill Equation, with coefficients:

$$
\begin{aligned}
& T_{0}=11.543, T_{1}=705.41, \\
& T_{2}=-19.532, T_{3}=15.338 .
\end{aligned}
$$

- $\quad z=[18.6,20.2]$ - Log-Normal Function:

$$
\begin{aligned}
& T_{r e}=T_{0}+T_{1} \exp \left[-\left(\frac{\ln \left(z / T_{2}\right)}{T_{3}}\right)^{2}\right] \text { with coefficients } \\
& T_{0}=16.488, T_{1}=34.915, T_{2}=17.003, T_{3}=0.082807 .
\end{aligned}
$$

- $\quad z=[20.2,21]-$ Sigmoidal Function, with coefficients:

$$
\begin{aligned}
& T_{0}=23.26, T_{1}=-8.5661, \\
& T_{2}=19.785, T_{3}=0.41168 .
\end{aligned}
$$

- $\quad Z=[21,22]-$ Log-Normal Function, with coefficients: 


$$
\begin{aligned}
& T_{0}=15.453, T_{1}=-0.54634, \\
& T_{2}=21.501, T_{3}=0.038693 .
\end{aligned}
$$

- $\quad Z=[22,30]-$ Log-Normal Function, with coefficients:

$$
\begin{aligned}
& T_{0}=60.399, T_{1}=-46.863, \\
& T_{2}=18.997, T_{3}=0.82554 .
\end{aligned}
$$

Finally, we report here below the redshift intervals and the corresponding analytic functions found in the case of the filtering model.

- $\mathrm{z}=[0,3.8]$-Polinomial Function of $8^{\circ}$ order, with coefficients:

$$
\begin{aligned}
& T_{0}=3794.4, T_{1}=6241, T_{2}=8613.8, \\
& T_{3}=-17286, T_{4}=14511, T_{5}=-6902.3, \\
& T_{6}=1923.6, T_{7}=-292.58, T_{8}=18.706 .
\end{aligned}
$$

- $z=[3.8,6.2]$-Sigmoidal Function, with coefficients:

$$
T_{0}=21591, T_{1}=-9256.6 \text {, }
$$$$
T_{2}=3.9293, T_{3}=0.50552
$$

- $\quad z=[6.2,10]-$ Log-Normal Function, with coefficients:

$$
\begin{aligned}
& T_{0}=52588, T_{1}=-51883, \\
& T_{2}=12.187, T_{3}=1.3666 .
\end{aligned}
$$

- $\quad z=[10,11]$-Decaying Exponential Function (expXOffset), with coefficients:

$$
\begin{aligned}
& T_{0}=-210.57, T_{1}=1989.4, \\
& T_{2}=1.8033, T_{3}=10 .
\end{aligned}
$$

- $\quad z=[11,15]$-Sigmoidal Function, with coefficients:

$$
\begin{aligned}
& T_{0}=4677.4, T_{1}=-4674.2, \\
& T_{2}=9.2859, T_{3}=1.2229 .
\end{aligned}
$$

- $\quad z=[15,18]$-Sigmoidal Function, with coefficients:

$$
\begin{aligned}
& T_{0}=180.89, T_{1}=-170.14, \\
& T_{2}=13.867, T_{3}=0.84978 .
\end{aligned}
$$

- $z=[18,21]$-Log-Normal Function, with coefficients:

$$
\begin{aligned}
& T_{0}=14.387, T_{1}=-2.5969, \\
& T_{2}=18.74, T_{3}=0.11421 .
\end{aligned}
$$

- $z=[21,30]$ - Log-Normal Function, with coefficients:

$$
\begin{aligned}
& T_{0}=8.1645, T_{1}=37.272, \\
& T_{2}=53.425, T_{3}=0.66903 .
\end{aligned}
$$

\section{B. Code Implementation in CAMB}

We give here some details on the routines we implemented in CAMB to make it able to reproduce the considered histories for the ionization fraction and electron temperature, summarizing the main innovations included in the modules of interest.

\section{B.1. Subroutine Modifications in Reionization Module}

The first improvement concern the type Reionization
Params with the inclusion of a new string variable, history, through which the user can discriminate between the models, stored in the settings parameters file params.ini.

The main function of the original module, Reionization_xe, has been now written for each history, Reionization_xeCF, Reionization_xeG, Reionization_xeL, Reionization_xeE. All of them retrieve the analytical ionization fraction for each redshift bin of interest.

With this approach is not necessary to parametrize $\chi_{e}$ in terms of the variable WindowVarMid:

$$
y=(1+z)^{3 / 2},
$$

where the exponent is set with the constant Reionization_zexp.

Some initial parameter values have been revisited too, such as the maximum redshift at which $\chi_{e}$ varies, fixed to 700 instead of 40 to take into account high redshift reionization phases, necessary for the early history, and the corresponding initial scale factor astart, inversely proportional to the redshift:

$$
a=\frac{1}{1+z}
$$

In the function Reionization timesteps the minimum number of time steps between tau start and tau_complete, the relevant times for the reionization process, has been incremented to 1000 , while for the implementation of the adopted ionization histories the functions listed below are no longer necessary:

- Reionization_doptdepth $(z)$, the subroutine that expresses the integral optical depth in terms of the scale factor,

- Reionization_GetOptDepth(Reion, ReionHist), the routine which evaluates the integral of the optical depth in the redshift interval $\left(0, z_{\max }^{\text {reio }}\right)$,

- Reionization_zreFromOptDepth(Reion, ReionHist), a general routine to find the $z_{r e}$ parameter given optical depth,

- Reionization_SetFromOptDepth(Reion, ReionHist), the subroutine that calculates the redshift of reionization.

This set of function, in fact, is related to the optical depth definition, i.e.

$$
\tau=\int_{0}^{\eta_{0}} \mathrm{~d} \eta a n_{e} \sigma_{T},
$$

as computed in the standard CAMB, while for the histories under examination we have implemented specific codes to evaluate it, both in our modified CAMB version and as independent codes.

\section{B.2. Subroutine Modifications in ThermoData Module}

The Thermodata module, implemented in modules. 990 source file, contains the subroutine inithermo(taumin, taumax), which evaluates the unperturbed baryon tem- 
perature and ionization fraction as function of time. If there is reionization, the function discriminates between the models, smoothly increases $\chi_{e}$ to the requested value and sets the actual_opt_depth to the value imposed by the corresponding model.

\section{List of Acronyms}

CMB: Cosmic Microwave Background

COrE: Cosmic Origins Explorer

WMAP: Wilkinson Microwave Anisotropy Probe

IGM: Inter Galactic Medium

SDSS: Sloan Digital Sky Survey

QSO: Quasar

IMF: Initial Mass Function
CAMB: Code for the Anisotropy Microwave Background

APS: Angular Power Spectrum

CF06: Suppression Reionization Model

G00: Filtering Reionization Model

CV: Cosmic Variance

SV: Sampling Variance

IR: InfraRed

ACT: Atacama Cosmology Telescope

ALMA: Atacama Large Millimeter/Submillimeter Array FWHM: Full Width Half Maximum

HFI: HFI Frequency Instrument

LFI: Low Frequency Instrument

CDM: Cold Dark Matter 